\title{
FEASIBILITY STUDY OF AN ACTIVE SYSTEM FOR TRANSFORMER NOHSE ABATEMENT
}

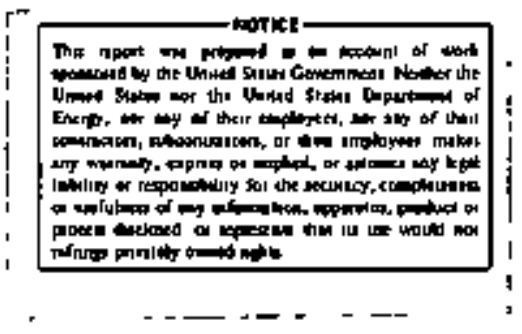

May 1979

\author{
Prepared by \\ Dean Yannucci \\ Westinghouse Electric Corporation \\ Medium Power Transformer Division \\ Sharon, Pennsylvania
}

Prepared for

U.S. Department of Energy

Assistant Secretary for Environment

Environmental Control Technology Division

Under Contract No, EE-77-C.02-4376 


\section{DISCLAIMER}

This report was prepared as an account of work sponsored by an agency of the United States Government. Neither the United States Government nor any agency Thereot, nor any of their employees, makes any warranty, express or implied, or assumes any legal liability or responsibility for the accuracy, completeness, or usefulness of any information, apparatus, product, or process disclosed, or represents that its use would not infringe privately owned rights. Reference herein to any specific commercial product, process, or service by trade name, trademark, manufacturer, or otherwise does not necessarily constitute or imply its endorsement, recommendation, or favoring by the United States Government or any agency thereof. The views and opinions of authors expressed herein do not necessarlly state or reflect those of the United States Government or any agency thereof. 


\section{DISCLAIMER}

Portions of this document may be illegible in electronic image products. Images are produced from the best available original document. 
Technical Raport Documentation Page

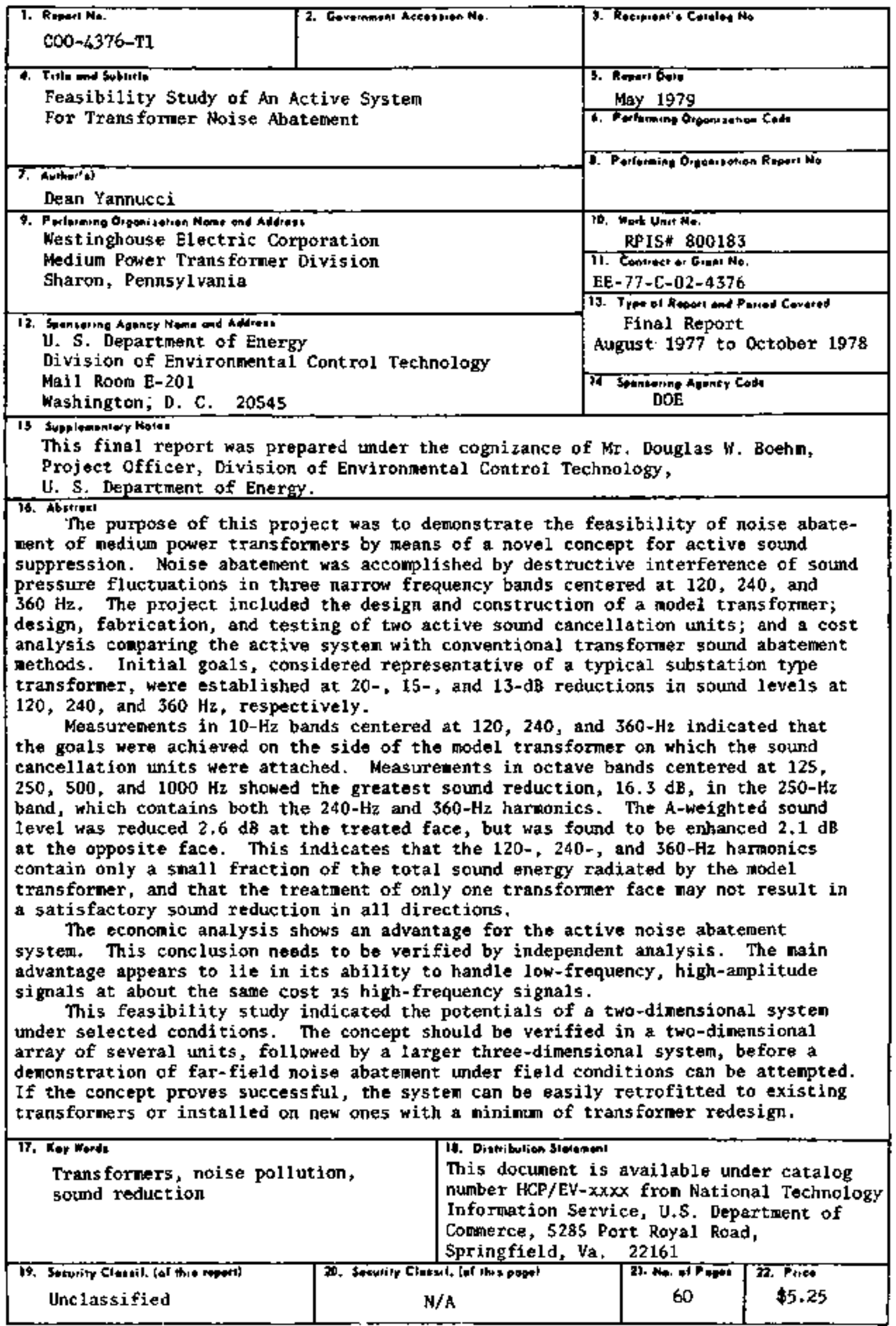




\section{ACKNOWLEDGMENT}

This report describes the results of a feasibility study for a nove] concept in active abatement of noise from a transformer. Many individuals contributed to the study and deserve special recognition. Dr. Allen Roger Davioson, Jr., performed, as part of his Ph.D. program at the George Washington University, the original basic research that is the cornerstone of the concept. Dr. Davidson and his colleagues at the Westinghouse Oceanic Division in Annapolis, Maryland, performed "bench-scale" testing and prepared the original proposal to the Department of Energy (DOE), then the Energy Research and Development Administration. Mr. David Keast of Bolt, Beranek and Newman, Inc., provided a useful perspective on the current transformer noise problem. Mr. Douglas W. Boehm, the DOE Project Officer, provided guidance and useful suggestions on the final report. Mr. Jit Vora of DOE and Dr. John Molino of the National Bureau of Standards provided comments on the draft report. Dr. Molino, in addition, conducted a brief psychoacoustic test on the effectiveness of the system. Finally, Dr. Russ Kevala and Ms. Barbara Breslin of The Aerospace Corporation, Germantown, Maryland, reviewed, edited, and prepared the final report for publication. 


\section{CONTENTS}

Page

EXECUTIVE SUMMARY

ix

I. INTRODUCTION

Current Practices for Noise Abatement of Transformers

Westinghouse Concept for Active

Noise Abatement

Objectives of Present Study

2

II. MODEL TRANSFORMER DESIGN 6

Design Objectives

Itentified Problem Areas

Alternative Solutions

III. ACTIVE NOISE ABATEMENT SYSTEM DESIGN \&

Broadband System

Narrow-band System

Checkout Testing

IV. TEST RESULTS OF MODEL TRANSFORMER AND

ACTIVE NOISE ABATEMENT SYSTEM

Test Objectives

Preliminary Testing

Effect of Variation of Applied Voltages

11

11

Radiation Pattern Tests

12

12

v. ECONOMIC STUDY 14

V1. CONCLUSIONS AND RECOMMENDATIONS 16

Conclusions

Recommendations

16

17

APPENDIX A. DETALLS OF THE MODEL TRANSFORMER DE\$IGN

A-1

APPENDIX B. DETAILS OF THE ACTIVE

NOISE ABATEMENT SYSTEA DESIGN

B-I 


\section{CONTENTS (Contimued)}

\section{Page}

APPENDIX C. COMPLETE TEST RESULTS

C-I

APPENDIX D. PSYCHOACOUSTIC DEMONSTRATION EXPERIMENT ON TRANSFORMER

NOISE REDUCTION

D-1 


\section{EXECUTTVE SUMMARY}

The purpose of this project was to demonstrate to the Environmental Control Technology Division of the U.S. Department of Energy the feasibility of noise abatement of medium power transformers by. means of a novel concept for active sound suppression. Noise abatement was accomplished by destructive interference of sound pressure fluctuations in three narrow frequency bands centered at 120,240 , and $360 \mathrm{~Hz}$. The following noise reduction goals were established for a 30-MVA, oil/air-cooled, 115-kV operating voltage, 450-kV basic impulse level (BIL) transformer, which was considered representative of a typical substation type transformer:

$\begin{array}{cc}\left.\text { Erequency ( } \mathrm{Hz}_{2}\right) & \text { Design Sound Level Reduction Goàl (dB) } \\ 120 & 20 \\ 240 & 15 \\ 360 & 13\end{array}$

The transformer spectrum typically exhibits "spikes" of 10 to $20 \mathrm{~dB}$ in magnitude at these three frequencies.

The project included the-design and construction of a model transformer; design, fabrication, and testing of two active sound canceliation units; and a cost analysis comparing the active system with other conventional transformer sound abatement method's. The model transformer configuration used an oil-immersed stacked core that represented a cross section of an actual transformer wall. The design goal for the model transformer was a $60-\mathrm{dB}(\mathrm{A})$ sound level at approximately 10 feet and a frequency spectrum similar to that from the reference 30-MVA transformer. The final design used a I-cubic-foot core inside an oil tank, with an outer housing of dimensions 3 feet by 2 feet by 1 foot.

After initial unsuccessful attempts at designing a broadband noise abatement system, a three-band system concentrating on frequency bands centered at 120,240 , and $360 \mathrm{~Hz}$ was developed. Preliminary laboratory tests conducted to verify the electronic design using a simulated sound source achieved the following sound reductions:

$\begin{array}{cc}\text { Frequency }(\mathrm{Hz}) & \text { Sound LeveI Reduction }(\mathrm{AB}) \\ & 20 \\ 240 & 17 \\ 360 & --\end{array}$

At $360 \mathrm{~Hz}$, the sound level reduction could not be measured because the initial sound level of the source at $360 \mathrm{~Hz}$ was below the ambient noise 
level. Each active sound "cancellation" unit consisted of a speaker-microphone assembly, electronic assembly, and d.c. power supply. A dynamic microphone sensed the local sound pressure about 4 inches from the transformer. The amplified and conditioned signal from the microphone was applied to a speaker that radiated a pressure wave out of phase with the local sound pressure fluctuations. The feedback from the speaker to the microphone and consequent instabilities were eliminated by an electronic feedback circujt with gain and phase controls. A stability analysis of the electromechanical system indicated a relative phase margin of $70^{\circ}$ and a gajn margin of $20 \mathrm{~dB}$.

Initial testing with the model transformer consisted of adjustments for optimum setting without self-oscillation. The transformer voltage was varied to check variation in output. The following sound-level reductions were achieved with transformer voltages of 120 and $130 \mathrm{~V}$ :

\section{Transformer Volts}

Frequency Band $(\mathrm{dB})$

$1 2 \longdiv { 0 \mathrm { Hz } } \underline { \underline { 2 4 0 \mathrm { Hz } } \quad 3 6 0 \mathrm { Hz } }$

21

20
37

25
19

13

Finally, broadband sound-level measurements were conducted at eight locations around the model transformer at a distance of 5.5 feet. Meastred sound reductions in octave bands and with $A$-wejghted and $C$-weighted filter settings were considerably smaller in magnitude than the narrow-band reductions. This is as expected, because the sound energy in the narrow bands around 120,240 , and $360 \mathrm{~Hz}$ is only a small fraction of the total sound energy radjated by the transformer. Even so, the annoyance caused by the transformer noise is expected to be reduced significantly because the puretone spikes at 120,240 , and $360 \mathrm{~Hz}$ would be significantly reduced.

Using internal Westinghouse Corporation data, the economic analysis determined additional capital costs for three noise abatement methods. The cost of noise abatement by reducing transformer core flux density was estimated to be $\$ 37,130$; by sound barriers, $\$ 59,778$; and by the present active method, $\$ 28,480$. These estimates have not been independently verified, however. The main advantage of the active system appears to be its abilty to handle low-frequency, high-amplitude signals at about the same cost as high-frequency signals.

The two-unit active system demonstrated in this study operated as a two-dimensional system. The design goals were achieved under selected conditions. A "three-dimensional" systern for a full-sized 30-MVA transformer is expected to require 120 units. Uncertainties about three-dimensional systems need to be resolved before far-field noise abatement under field conditions can be demonstrated. If the concept proves successful, the system can be easily retrofitted to existing transformers as well as installed on new ones with a minimum of transformer redesign. 


\section{INTRODUCTION}

\section{CURRENT PRACTICES FOR NOISE ABATEMENT OF TRANSFORMERS}

Substation noise, most notably the "hum" from transformers and reactors," has been a major cause of noise complaints recejved by electric utilities in the United States. Complaints have resulted from transformer noise levels as low as $36 \mathrm{~dB}(A) *$ outside residences in quiet neighborhoods. It has been estimated that over 2.5 million people in the United States now live at locations where substation noise exceeds $40 \mathrm{~dB}(\mathrm{~A})$.

Bolt, Beranek and Newman, Inc., an acoustical consulting firm, has estimated that U.S. electric utilities currently spend over $\$ 100$ million per year for transformer noise abatement. At least one utility always purchases special low-noise transformers because its installations are in populated areas. Another, for the same reason, always installs masonry enclosures for its transformers. Other utilities, serving less populated areas, site their substations to avoid proximity to residences and may purchase large areas of buffer land.

Reducing transformer noise is generally well within the capability of current abatement technology. Vendors can supply special low-noise power transformers with noise levels as much as 20 to $35 \mathrm{~dB}(\mathrm{~A})$ below the National Electrical Manufacturers' Association standard noise rating at a capital cost markup of about 2 percent per $d B$ ( 40 percent for $20 \mathrm{~dB}$ ). Masonry barrier walls around transformers, also guite costly, can reduce noise by 10 to $15 \mathrm{~dB}(\mathrm{~A})$; full enclosures car yjeld up to $25 \mathrm{~dB}$ reduction in A-weighted sound levels. The engineering and construction costs of such structures are, however, quite significant, and in addition to these large capital investments, there can be additional operating costs:

- Reduced electrical efficiency because of greater copper losses in large-core, low-noise transformers;

- Reduced cooling efficiency because of enclosing structures; and

- Restricted maintenance access from the enclosing structures.

\footnotetext{
* The noise from an iron-core device, such as a transformer or reactor, is produced primarily by magnetostrictive motion of the core. Secondarily, noise can be produced by cooling fans used on some transformers and reactors.

** $d B(A)$ is the $A$-weighted sound level using a logarithmic scaie with a reference sound pressure of 20 micronewtons per square meter. Explanations of acoustical engineering terminology are presented in Handbook of Noise Measurement, Seventh Edition, by A.P.G. Peterson and E.E. Gross, Jr., ¿enerâl Radio Co., Concord, Massachusetts, 1972.
} 
Although existing noise abatement techniques are probably adequate to reduce transformer noise to acceptable levels in almost all current situations, future scenarios are unknown. Furthermore, there may be a need for research into novel techniques to reduce the attendant cost and operational inefficiencies of present substation noise abatement techniques.

\section{WESTINGHOUSE CONCEPT FOR ACTIVE NOISE ABATEMENT*}

The special low-noise transformers and masonty barriers mentioned above are considered passive abatement techniques. Because audible sound from transformers has discrete frequency components $(120,240,360 \mathrm{~Hz}$, etc.), there is good possibility of reduction of the sound through an active technique of destructive interference. The technique converts the acoustic radiation from a vibrating surface into electrical signals so that the signal can be conditioned and amplified to frive a sound projector in phase opposition to the source radiation. The net acoustic volume change is then driven to a null around the vibrating surface by destructive interference. The acoustic radiation is thus inhibited from propagating into the far field,**

The first known patent for this technique was granted in 1939 to lueg for the concept of using a microphone in front of an acoustical source and triving a speaker in phase opposition to the signal detected in order to reduce the sound intensity. Probably the first attempt to use an active noise abatement system was made by Olson and May in 1953 during their research on an Electronic Sound Absorber. Here again, a microphone, amplifier, and loud-speaker were employed to produce equal-amplitude, opposite phase acoustic signals to cancel sound in a local area. This device was proposed for use in noisy environments (inside aircraft, near machines, etc.) where it was desirable to reduce noise levels that were uncomfortable to people present. Some degree of success was achieved, but only in a very restrictive area around the microphone. More recently, Westinghouse developed an active nojse abatement technique as part of its general sound reduction research program; Figure 1 is a diagram of this technique. Where other systems in the past have failed, this technique has succeeded by using a feedback and control design to compensate for changes in the transformer environment or applied voltage.

The actual application of the sound cancellation system to mediumsized transformers*** will require an array of individual units mounted on

\footnotetext{
* Material for this section has been derived from "Active Reduction of Low Frequency Sound From Large Vibrating Structures," A.R. Davidson, Dactoral Thesis, School of Engineering and Applied Science of The George Washington University, May 1975 and "Transformer Noise Reduction System," Westinghouse proposal to the Department of Energy, July 29, 1976.
}

\footnotetext{
* Roughly, the acoustic far field is the region that is at least one sound wavelength away from the transformer.

*** For this report, medium size is assumed to be the range of 5 to 200 MVA.
} 
the transformer walls, (Figure 2). The individual sound reduction units will contain a speaker, microphone, power supply, audio power amplifier, gain and phase shaping circuits, and various required delay circuits. The enclosure of each unit also functions as a speaker enclosure. The structural design is an integral part of the phase and gain control system; internal damping and baftling are required to provide a flat phase and gain characteristic over all the frequencies of interest. The microphone must also have a flat response over that frequency range. The individual units must be sealed against the weather elements, but must be able to "breathe" in order to equalize internal and external pressures. This requirement can be accomplished by using metal conduit or transformer tank wall braces which can house the power supply conductors. This type of conduit is commonly used to house control wire for fans, pumps, and alarms.

When mounted on the transformer, the units in an array individually interfere with the sound radiation from a particular area; all of the units collectively reduce the sound on the whole area. The advantage of this technique of noise abatement over passive systems is in the simplicity of application to either retrofitting present transformer installations or incorporation into future designs. Another advantage is that if one unit fails, the other units will automatically readjust so that total performance of the system will degrade only imperceptibly.

Westinghouse originally proposed to investigate the application of this technique to reducing low-frequency audible noise from large transformer installations. The first phase of the proposal was to assess the feasibility of the concept by demonstration on a model transformer and a limited number of optimized active sound cancellation wits. The second phase proposed the fabrication of a number of units to be mounted and tested on an actual medium power transformer to measure the effectiveness of the system in an outdoor setup. It was decided, after discussions with the Department of Energy, to undertake the preliminary feasibility study. The results of that study are presented in this report.

\section{OBJECTIVES OF PRESENT STUDY}

The major objective of the technical work described in this report was to demonstrate the feasibility of reducing the sound from a model transformer by means of an active noise abatement system. An economic analysis was provided to support the feasibility study. The specific objectives were to

- Design and construct a small model transformer;

- Design and procure two prototype sound cancellation units for the model transformer;

- Assemble and demonstrate an active noise abatement system for the model transformer to a performance level (as measured in the far field) of at least $20 \mathrm{~dB}$ reduction of sound for the $120-\mathrm{Hz}$ 


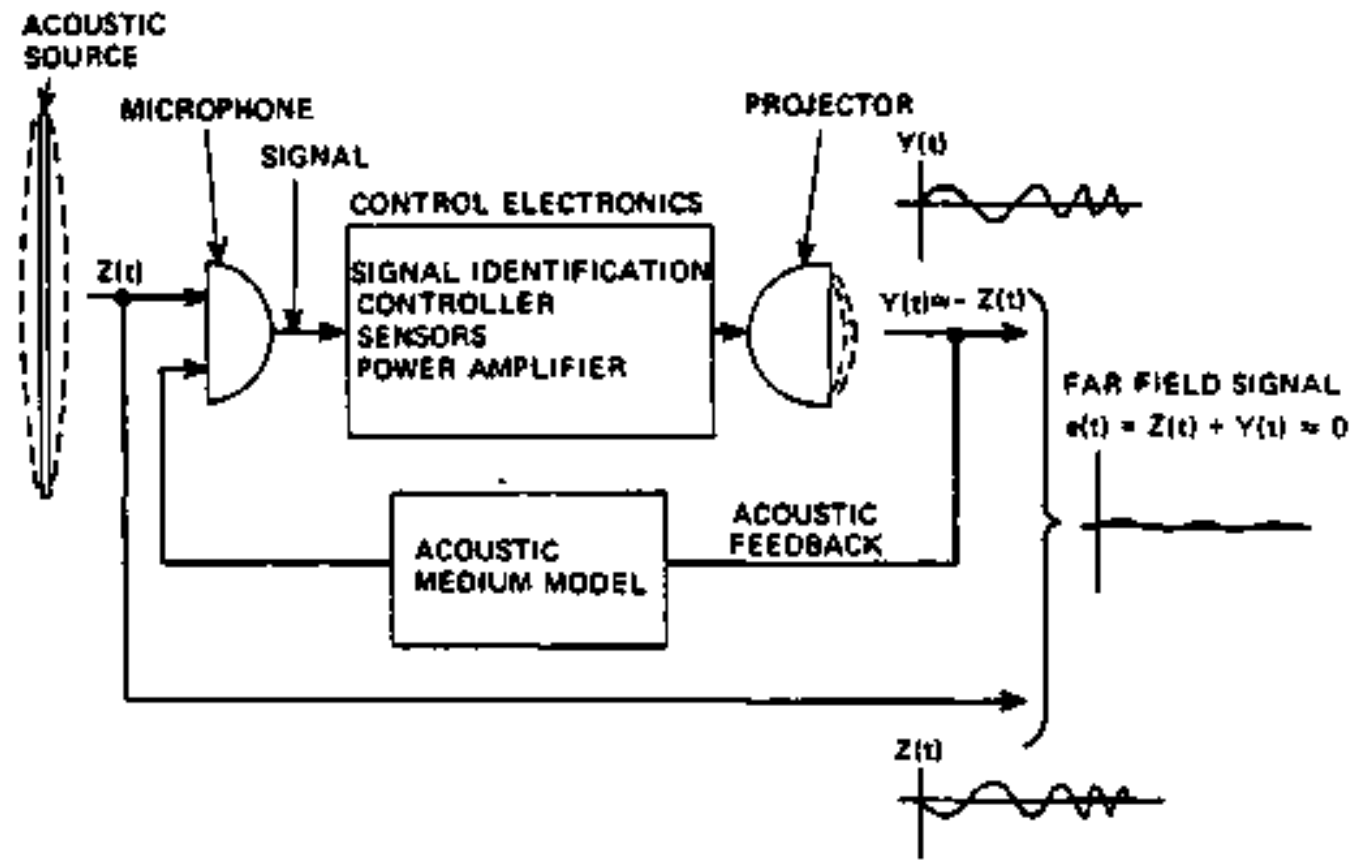

Figure 1. Noise Abatement System

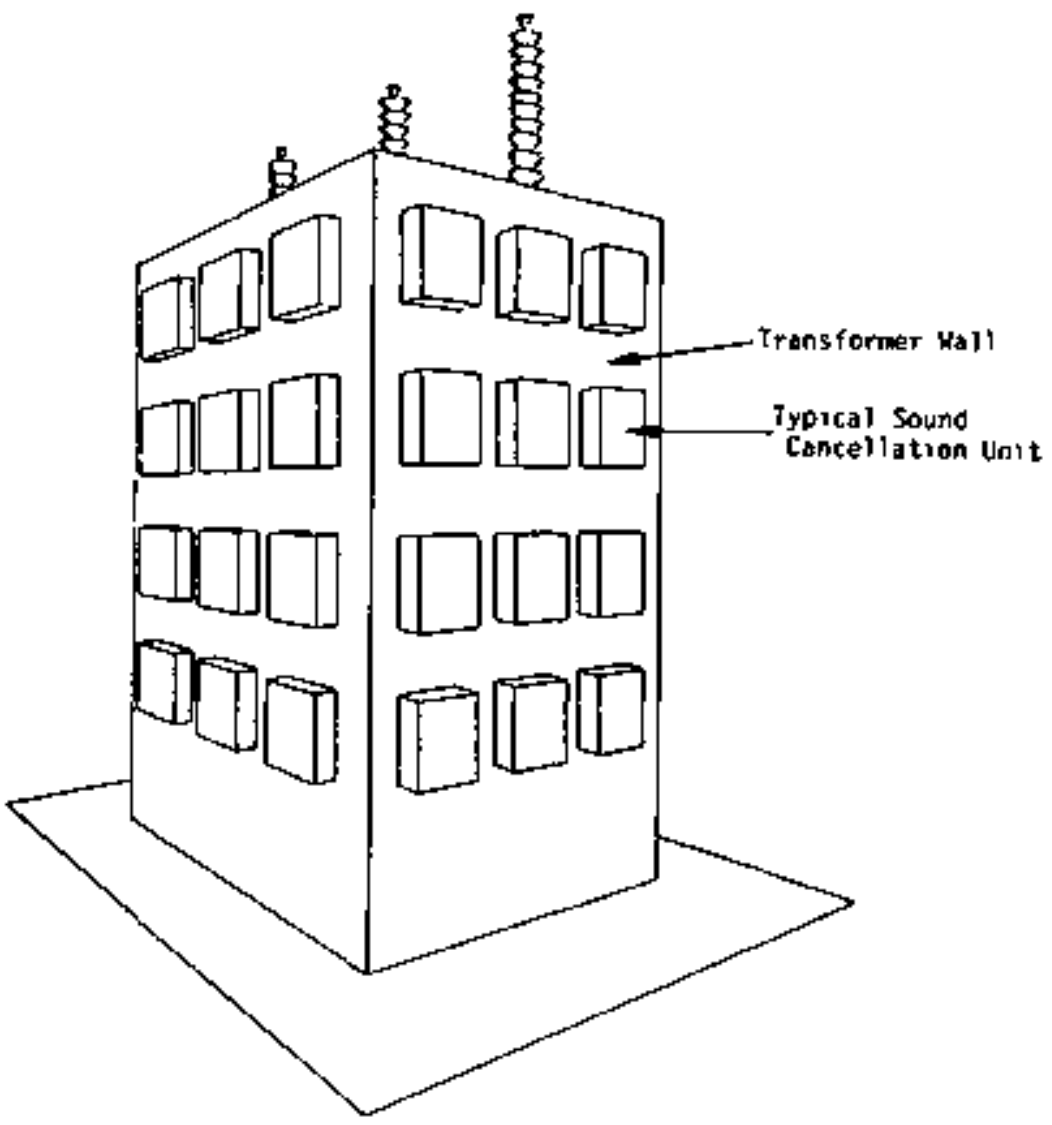

Figure 2. Installation of an Active Noise Abatement System Onto a Transformer 
component, $15 \mathrm{~dB}$ for the $240-\mathrm{Hz}$ component, and $13 \mathrm{~dB}$ for the $360-\mathrm{Hz}$ component;

- Submit a complete engineering diagram of the system design and the resuts of the testing in an anechoic chamber; and

- Perform an economic analysis comparing the active noise abatement system with other systems for a 30-MVA, oil to air (OA), $115-\mathrm{kV}$ operating voltage, $450-\mathrm{kV}$ basic impulse level (BIL) rated transformer. 


\section{MODEL TRANSFORMER DESIGN}

\section{DESIGN. OBJECTIVES}

The primary objective of the model transformer design was to obtain a sound level of $60 \mathrm{~dB}(\mathrm{~A})$ at approximately $0.3 \mathrm{~m}$ ( $1 \mathrm{ft}$ ) from the model in an anechoic chamber with an acoustically reflective floor. The following narrow-band spectral characteristics at line frequency harmonics were also deemed to be desirable:

Frequency (Hz) Sound Pressure Level dB (10- $\mathrm{Hz}$ bandwidth)

$\begin{array}{cc}120 & 76 \\ 240 & 54-59 \\ 360 & .43-51\end{array}$

Sound energy at frequencies above $360 \mathrm{~Hz}$ was to be kept at least $26 \mathrm{~dB}$ below that contained at frequencies under $360 \mathrm{~Hz}$. These sound levels and spectral characteristics were judged to be representative of a 30-MVA oil/air substation transf ormer.

\section{IDENTIFIED PROBLEM AREAS}

The design of the model transformer posed severa] specific problems. It was difficult to meet the Jow-frequency sound level requirements with a model transformer in a box with a $0.3-\mathrm{m}^{3}$ ( $1 \mathrm{ft}^{3}$ ) volume. Surfaces with dimensions that are small compared to the wavelength of sound radiate very inefficiently at and below the frequency corresponding to the wavelength.* Conversely, high-frequency sound from the model will be radiated much more efficiently than that from the full-sized transformer. Finally, it was determined that it is virtually impossible to obtain sound abatement from more than one face of the cube, using two cancellation units as planned. The carcellar unit has to be located within $\lambda / 3$ of the sound-producing surface to be effective.

\section{ALTERNATIVE SOLUTIONS}

Two configurations were evaluated. The first is a large enclosure with dimensions of $0.91 \mathrm{~m}$ by $0.61 \mathrm{~m}$ by $0.305 \mathrm{~m}$ ( $3 \mathrm{ft}$ by $2 \mathrm{ft}$ by $1 \mathrm{ft}$ ), housing a * The wavelength $(\lambda)$, frequency (f), and velocity (c) of sound are related by the equation:

$$
\lambda=c / f
$$

Because the speed of sound is fixed at a given temperature, the wavelength and frequency are inversely related. For a $1-\mathrm{ft}^{2}$ surface, radiation efficiency would begin to top off below $1000 \mathrm{~Hz}$. 
core and coil assembly of about $0.03 \mathrm{~m}^{3}\left(1 \mathrm{ft}^{3}\right)$ volume. The enclosure would permit sound to radiate from the $0.305 \mathrm{~m}$ by $0.61 \mathrm{~m}$ ( $1 \mathrm{ft}$ by $2 \mathrm{ft}$ ) open face, while preventing radiation from the other faces. Sound cancellation units would be piaced on the open face side. The second configuration is a simulated transformer source in a $0.03-\mathrm{m}^{3}\left(1-\mathrm{ft}^{3}\right)$ enclosure. The enclosure would have four rigid (nonradiating) sides radjating sound from two opposite faces on which the sound cancellation units would be located. The source of sound may be speakers $\alpha$ a transformer with a core gap.

The use of a transformer case would permit the core to be immersed in an oil medium. It was recognized that the case would have to be modified to attenuate the undesirable sound radiation above $360 \mathrm{~Hz}$. The sound level of the model transformer could have been increased by varying the clamping forces to overstress the core material or by using air gaps in the core. Nejther of these conditions exist in the actual transformer core, however, so it was decided not to use them for the model.

The first configuration was selected. Details of the design of the model transformer are provided in Appendix A. 


\section{II]. ACTIVE NOISE ABATEMENT SYSTEM DESIGN}

\section{BROADBAND SYSTEM}

A broadband phase inversion circuit was designed and incorporated in breadboard form, and commercial speakers were modifjed to lower their resonant frequency. Initial tests were performed to determine the system response characteristics, which were then used for the design of the feedback cancellation and the compensation networks to "linearize" the speaker response.

The broadband system used a dynamic microphone, which was located inside the speaker cabinet, to sense the local sound. Unfortunately, the speaker and microphone did not perform as expected from theory. The result was a response that was ronlinear, inconsistent, and unpredictable. After several unsuccessful attempts to find some broadband method of compensation, a serjes of measurements were made. It was concluded that

1. Uniform spreading loss occurs from about $2.5 \mathrm{~cm}$ (I in.) to $2.5 \mathrm{~m}$ (3 ft) from the speaker.

2. A hand-held microphone in front of the system and the microphone in the speaker cabinet show significantly different signal magnitudes and phase, indicating an acoustical path through the speaker cabinet to the system microphone.

3. The speaker produces noticeable distortion below $90 \mathrm{~Hz}$, but the distortion was not detectable above $120 \mathrm{~Hz}$.

The first conclusion could possibly have worked to an advantage when combined with the second, because it should simplify the far field transfer function for at least the lower frequencies. The third conclusion was more serious because it indicated the need for either scrapping the existing speakers for ones with more rigid cone mounting and repeating the process of lowering the resonant frequency, or generating a new set of transfer functions and obtaining the electronics to simulate them.

\section{NARROW-BAND SYSTEM}

Another alternative was to abandon the broadband approach and concentrate on the three frequencies of interest $(120,240$, and $360 \mathrm{~Hz})$, ignoring higher frequencies. This approach would provide a means of tailoring the system response to each frequency without affecting the response to the other frequencies. The basic theory of active noise cancellation is unchanged but, because of the unique spectrum of the transformer audio output, it is possible to obtain the necessary system response while increasing the number of electronic components by 10 or 15 percent. This added cost may be offset by a simpler adjustment procedure using a sufficient number of noninteracting adjustments. This approach was also the most promising in view of 
the program schedule and funding. The following conclusions were reached:

- Abandon the broadband approach.

- Design, fabricate, and test a three-band system with the bands centered at 120,240 , and $360 \mathrm{~Hz}$, using the same speakers and electronic stages.

- Change the location of the system microphone to $10.2 \mathrm{~cm}$ (4 in.) from the sound source.

- Include feedback cancellation to eliminate any possibility of high frequency "squeals."

\section{CHECKOUT TESTING}

A simulated test source, using a small speaker, was designed and fabricated to the approximate dimensions of the model transformer. The radiating surface was $\mathrm{J} / \mathrm{R}$-in. plexiglass. Two amplifiers were fabricated and checked out in the laboratory. Each had separate adjustments for phase and amplitude gains for each of the three frequencies: 120,240 , and $360 \mathrm{~Hz}$. The microphone on each speaker was moved to the outside of the enclosure, about 2 in. back from the front face of the speaker (Figure 2), which places the microphone about $4 \mathrm{in}$. $(10.2 \mathrm{~cm})$ from the sound source.

Tests were conducted in the laboratory and outdoors using the simulated sound source. The sound reductions measured outdoors were slightly lower than those obtained in the laboratory, but still exceeded the goals, as indicated below.

\begin{tabular}{ccc} 
Frequency & \multicolumn{2}{c}{ Sound Leve] Reduct ion } \\
\cline { 2 - 3 } 120 & Design & Actuald(dB) \\
240 & 20 & $20+$ \\
360 & 15 & 17 \\
& 13 & $\ldots$
\end{tabular}

No usable information could be obtained at $360 \mathrm{~Hz}$ because of a very deep nulJ in the response of the simulated sound source, which reduced its output below ambient. The amplifiet gain and phase characteristics at $360 \mathrm{~Hz}$ are similar to those at 120 and $240 \mathrm{~Hz}$, indicating that the system was operating as designed. Because there were no indicatjons that operation at $360 \mathrm{~Hz}$ was not possible, it was decided that it would not be necessary to modify the sound source just to prove satjsfactory operation at that frequency.

Flexibitity was designed into all of the filters so that the amount of control could be adjusted to meet the specific application requirements. The feedback was also checked both in the laboratory and outdoors and was found 
to be lower during the outdoor tests. The differences between the quieting and feedback responses in the laboratory and outdoors are attributed to the differences in impedences to the miçrophones and speakers from the two testing conditions. Details of the actual design of the cancellation units are provided in Appendix $\mathrm{B}$. 


\section{V. TEST RESULTS OF MODEL TRANSFORMER AND ACTIVE NOISE ABATEMENT SYSTEM}

\section{TEST OBJECTIVES}

Tests were conducted on the model transformers and the active noise abatement system in the Westinghouse Sharon Division sound room. The room measures about $50 \mathrm{ft}(15 \mathrm{~m})$ in all dimensions and has a concrete floor, chosen because it more properly simulates the conditions of a transformer installation. The walls and ceiling are lined with arechoic fiberglass wedges.

The instrumentation used to gerform the sound tests included the following:

- General Radio Sound Level Meter, Type 1933

- Quan-Tech Model 304A Analyzer

- Houston Instrument Series 2000 Omnigraphic X-Y Recorder

This instrumentation permitted sound-level measurements with A-weighting and C-weighting, in octave bands, and in $10-\mathrm{Hz}$ bands centered at any frequency. The model transformer was energized through a Variac so that the excitation voltage could be adjusted independently of the canceller units.

The transformer, except for one end, was completely enclosed in a sound enclosure box. Sound radiation is principally from this open end, and the two cancellation units were mounted on this end. The housing was placed on a table, about $76 \mathrm{~cm}$ ( $30 \mathrm{in.}$ ) above the floor. The testing was done in three stages, with the following objectives:

- Preliminary testing to perform optimum adjustments and prevent self-oscillations,

- Variation of applied voltage to determine output variation from applying different voltages, and

- Noise radiation pattern tests to determine spatial variation in per formance.

\section{PRELIMINARY TESTING}

With the model transformer energized, each cancellation unit was adjusted for optimum cancellation of the $120-, 240-$, and $360-\mathrm{Hz}$ components of the transformer sound. The procedure consisted of adjustments to the electronics of the cancellation units so that each wit would produce 
sound out of phase, but equal in magnitude, to the transformer sound. Also, to prevent self-oscillations, adjustments were made so no feedback occurred in the control signal loop of the amplifier. After the units were properly adjusted, no further adjustiments were made except to improve performance at $120 \mathrm{~Hz}$. The amount of $120-\mathrm{Hz}$ sound from the transformer was initially low, about $7 \mathrm{~dB}$ above the ambient, and further adjustments were required.

\section{EFFECT OF VARIATION OF APPLIED VOLTAGES}

To insure that variations in voltage did not affect the active noise cancellation system, two tests were conducted. First, four different voltages ranging from $100 \mathrm{~V}$ to $130 \mathrm{~V}$ were applied to the transformer, and the resulting sound levels were measured. Second, two different voltages were applied with the sound cancellation wits on and off, and the sound reductions were measured. The results of the first test for the $120-\mathrm{Hz}$ component are listed below.

$\begin{array}{cc}\text { Voltage }(V) & 120-\mathrm{Hz} \text {. Sound Level at } 1 \mathrm{~m}(3 \mathrm{ft}) \\ 100 & 58 \\ 110 & 59 \\ 120 & 57.5 \\ 130 & 55.5\end{array}$

The following are the results of the second test.

\begin{tabular}{|c|c|c|c|c|}
\hline Transf ormer & \multicolumn{4}{|c|}{ Sound Reduction ( $\mathrm{dB})$} \\
\hline Voltage & $120 \mathrm{~Hz}$ & $240 \mathrm{~Hz}$ & $360 \mathrm{~Hz}$ & $480 \mathrm{~Hz}$ \\
\hline $\begin{array}{l}120 \\
130\end{array}$ & $\begin{array}{l}21 \\
20\end{array}$ & $\begin{array}{l}37 \\
25\end{array}$ & $\begin{array}{l}19 \\
13\end{array}$ & $\begin{array}{l}0 \\
0\end{array}$ \\
\hline
\end{tabular}

It was concluded that variations in applied voltage did not result in a significant difference in either the sound levels produced by the model transformer or the performance of the active noise abatement system. The complete results are presented in Appendix C.

\section{RADIATION PATTERN TESTS}

Measurements were taken at eight locations equally spaced in a circle with a radius of $1.7 \mathrm{~m}(5.5 \mathrm{ft})$ around the model transformer. The purpose of these measurements was to determine a radiation pattern for the model transformer in the Jarge ( $3 \mathrm{ft}$ by $2 \mathrm{ft}$ by $1 \mathrm{ft}$ ) enclosure and to evaluate the overall effectiveriess of the two-unit active noise reduction system. Figure 3 shows the measured sound levels with and without the canceller units operating. The A-weighted sound levels at $1.7 \mathrm{~m}(5.5 \mathrm{ft})$ were $6 \mathrm{~dB}$ higher in the 
direction of the open enclosure face (front face) than in the opposite direction. The noise reduction system lowered the decibel (A-weighted) level by $2.6 \mathrm{~dB}$ at the front face but enhanced the level by $2.1 \mathrm{~dB}$ at the opposite face. Measurements in octave bands centered at 125, 250, 500 and $1000 \mathrm{~Hz}$ showed the greatest sound reduction, the maximum being $16.3 \mathrm{~dB}$ in the $250-\mathrm{Hz}$ band. This is not surprising because the $250-\mathrm{Hz}$ octave band spans 180 to $355 \mathrm{~Hz}$ and thus contains both the $240-\mathrm{Hz}$ and $360-\mathrm{Hz}$ total components. The complete results of the radiation pattern tests are presented in Appendix $C$.

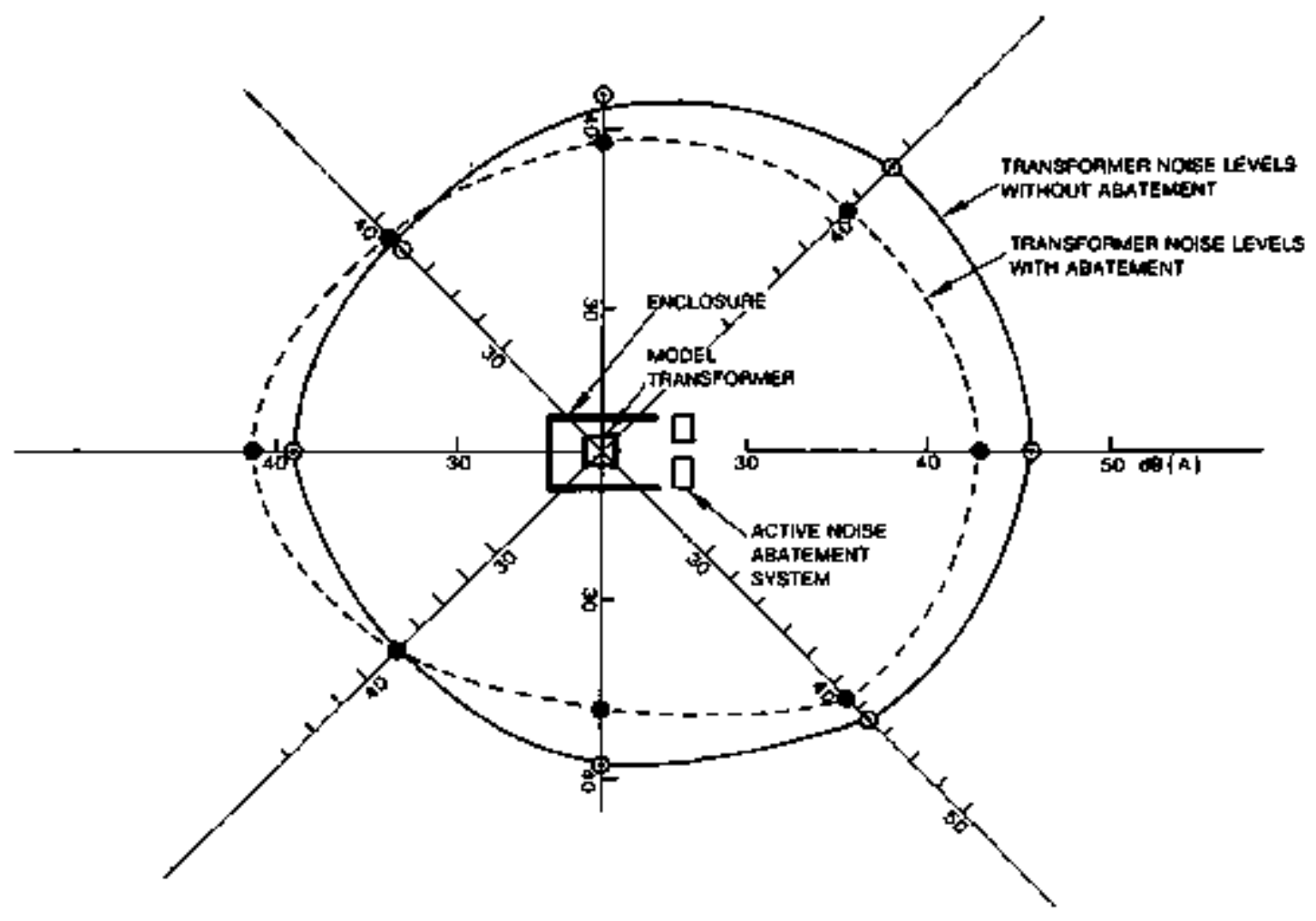

Figure 3. Measured Sound Levets Around Model Transformers at a Distance of $1.7 \mathrm{~m}(5.5 \mathrm{ft})$ 


\section{v. ECONOMIC STUDY*}

There are currently two fundamental approaches to the reduction of transformer noise. The first method is to reduce the operating flux density of the transformer core. This is the more effective and less costly method for large transformers. A change of 10 percent in the operating flux density will result in a 2- or 3-dB reduction in the noise level. Thus, for a 20-dB noise reduction, the core weight has to be increased by approximately 100 percent to maintain the same electrical output from the transformer.

The second method involves the use of sound barriers, either mounted on the transformer or in its proximity. On new transformers, panels may be built over the outside of the regular transformer tank in the factory. These panels are made of $3.18-\mathrm{mm}(0.125-\mathrm{in}$.) thick steel, and about a $15.2-\mathrm{cm}$ (6-in.) space is allowed between them and the transformer wall. The space is partially filled with sound-absorbing material, such as glass fiber, and larger cooling radiators would be needed to compensate for the reduced heat transfer of the tank wall. The installation of panels in the field on units not designed for thern is a more complicated and expensive procedure. Barriers external to the transformer may take the form of complete enclosures, roofless enclosures, and partially surrounding walls. To be effective, the enclosure must have a low sound transmission coefficient, which requires appreciable mass per unit surface area. The effectiveness of enclosures can be enhanced by lining with a material of high abrosption coefficient to prevent sound buildup through multiple reflectors. Roofless or partial enclosures are less effective, particularly in the far field, because of diffraction at the edges. Additional economic penalties and engineering complexities are introduced by the need for additional acoustic sealing for incoming electrical lines, and cooling requirements.

For this economic analysis, three cases will be assumed: (1) the flux density method, (2) the sound barrier method, and (3) the active noise abatement system described in this report. The reference design requirement is a $20-\mathrm{dB}$ noise reduction for a 30 -MVA, $115-\mathrm{kV}$ operating, $450-\mathrm{kV}$ basic impulse level transformer. The transformer has a length of $3.66 \mathrm{~m}$ (12 ft), with a width of $5.05 \mathrm{~m}$ (16.6 ft), and a height over bushings of $5.05 \mathrm{~m}$ (16.6 ft). For this analysis, 1975 dollars are assurned.

For the flux density method, a 100-percent increase in the core or about an addjtional $3046 \mathrm{~kg}(19,900 \mathrm{lb})$ will be required, with an attendant $\$ 1,000$ cost penalty for handling and transportation. There will be a credit due to reduced no-load losses, estimates at $\$ 1,200$ per kilowatt, which reduces the capital cost by $\$ 13,000$. The net additional capital cost for this method is $\$ 37,130$.

\footnotetext{
* Actual figures used in this study were derived from an interna! Westinghouse study.
} 
For the sound barrier method, the cost is quite variable. The barrier cost for a $20-\mathrm{dB}$ reduction is estimated to be $\$ 59,778$, assurning $\$ 1,476$ per meter of barrier; 40.5 meters are required.

For the active noise abatement system, it has been estimated that 120 cancellation units would be required for a $20-\mathrm{dB}$ reduction. The additional total capital cost is estimated to be about $\$ 28,480$, based on $\$ 175$ per unit (in quantities of 10,000 units per year). The additional operating and maintenance cost is estimated to be $\$ 2,000$ over the lifetime of the transformer.

To summarize, the estimated additional capital costs are listed below.

Noise Reduction Method

F Jux Density

Sound Barrier

Active Noise Abatement
Additional Capital Cost in 1975 Dollars

37,130

59,778

28,480 


\section{CONCLUSIONS AND RECOMMENDATIONS}

\section{CONCLUSIONS}

The following conclusions were arrived at from this study:

- The large enclosure design for the model transformer is selected because

-- It provides an adequate over-all noise level,

-- The high-frequency spectrum can simulate the spectrum of a medium-sized transformer,

- The radiating surface is not greater than the far field $(e . g+, \lambda / 3)$ restriction, and

-- The configuration provides for an oil-immersed transformer with a stacked core, representing the cross section of an actual transformer wall.

- The active noise abatement system design is adequate, considering the following factors:

-- The system uses two canceliation units; a 30-MVA transformer would requite 120 cancellation units;

-- The system was a "two-dimensional" model; a 30-MVA transformer would require a "three-dimensional" solution;

-- The narrow-band system had center frequencies at 120, 240, and $360 \mathrm{~Hz}$; and

- The relative stability of the electromagnetic system, with a phase margin of $70^{\circ}$ and a gain margin of $20 \mathrm{~dB}$, is adequate.

The testing program indicates that

-- Preliminary checkout test of the system shows sound reduction exceeding design goals for 120 - and $240-\mathrm{Hz}$ components; however, the $360-\mathrm{Hz}$ component could not be measured because of low output from the transformer and high ambient noise;

-- Cancellation of feedback signal is adequate; 
-- Variation in the applied voltage to the transformer does not significantly affect either the transformer output sound or the performance of the active noise abatement system; and

-- The noise radiation pattern test, using A-weighted, Cweighted, and octave band measurements shows that the overall $A$-weighted sound level is reduced by $2.6 \mathrm{~dB}$ and the $250 \mathrm{~Hz}$ octave band level is reduced by $16.3 \mathrm{~dB}$ in the direction of the face on which the sound cancellation units were attached. There is a slight reinforcernent of sound in the opposite direction.

- The active noise abatement system appears to work equally well at the low-frequency end as at the higher frequencies, unlike the conventional sound barrier system.

o The economic analysis shows an advantage for the active noise abatement system; however, this conclusion cannot be verified by independent analysis.

\section{RECOMMENDATIONS}

More detajled research is needed to determine the interactions of constructive and destructive interference to ensure proper noise abatement in the far field. Specifically, a two-dimensional array of several (perhaps 10) units to simulate the full side of the medium transformer should be built and tested. Measurements should be taken at several locations.

A marketing survey should be performed to better define such parameters as the design goals for sound abatement for both new and operating transformers; the number, Jocation, and schedule for retrofitting operating transformers; and better cost estimates of this system over other abatement systems.

Research and development for the active noise abatement system should be considered a long-term project. Many uncertainties should be resolved before undertaking Phases I, II, and II] for a full-sized demonstration as originally proposed by Westinghouse.*

* Westinghouse proposal to the Department of Energy, "Transformer Noise Reduction System," July 79 , 1976. 


\section{APPENDIX A. DETAILS OF THE MODEL TRANSFORMER DESIGN}

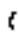

\section{CORE DESIGN}

The model transformer used a stacked, laminated, "D" type core used in the power transformer industry. The ends of each lamination were sheared at a $45^{\circ}$ angle. The core material was "M-5" grade electrical steel $0.305 \mathrm{~mm}$ (0.012 in.) thick so that the flux accurred cross-grain to the material, which resulted in increasing the noise level of the model transformer such that the 60-dB(A) level could be obtained. Figure A-1 shows the individual yoke and leg punching dimensions. Figure $A-2$ shows the core assembly. The overall stack height of the core was $44.45 \mathrm{~mm}$ (1.75 in.). The losses of the model core with the excitation winding energized are summarjzed below.

\begin{tabular}{|c|c|c|c|}
\hline $\begin{array}{l}\text { Primary } \\
\text { Applied } \\
\text { Voltage } \\
(V) \\
\end{array}$ & $\begin{array}{c}\text { Winding } \\
\text { Current } \\
\text { (A) }\end{array}$ & $\begin{array}{c}\text { True Watt } \\
\text { Loss } \\
\text { (W) }\end{array}$ & $\begin{array}{c}\text { Core } \\
\text { FJux Oensity } \\
(T)\end{array}$ \\
\hline $\begin{array}{l}90 \\
100 \\
110 \\
120 \\
125 \\
128.7\end{array}$ & $\begin{array}{r}.83 \\
1.18 \\
2.90 \\
5.60 \\
11.80 \\
15.00\end{array}$ & $\begin{array}{l}23.1 \\
28.9 \\
39.0 \\
55.0 \\
78.0 \\
98.0\end{array}$ & $\begin{array}{l}1.1596 \\
1.2885 \\
1.4173 \\
1.5462 \\
1.6106 \\
1.6582\end{array}$ \\
\hline
\end{tabular}

It should be noted that the flux density at the nominal voltage rating of the transformer, $120 \mathrm{~V}$, is $1.346 \mathrm{~T}$. This operating flux density was obtained because the core was designed and built with oross-grain electrical steel. The core was stacked and the legs taped with $0.254-\mathrm{mm}(0.0 \mathrm{I}-\mathrm{in}$.) cotton tape. The taped leg and all exposed edges of the yoke were resin bonded to reduce high-frequency noise.

\section{COIL DESIGN}

Two turns of $0.254 \mathrm{~mm}(0.0 \mathrm{l}-\mathrm{in}.) \mathrm{Kraft}$ paper was wrapped over each core leg. Round copper wire $2.06 \mathrm{~mm}$ (0.081 in.) in diameter was used for the winding conductor; it was coated with quad enamel (Westinghouse PDS \# 612JJAR) and 76 turns were wound on each core leg for a total of 152 turns. The total length of the wire was $33.53 \mathrm{~m}(110 \mathrm{ft})$ and weighed $1 \mathrm{~kg}$ (2.2 Ib). The start and finish leads were taped with $0.178-\mathrm{mm}(0.007-\mathrm{in}$. cotton tape to protect them from abrasive actions. The outside of the coil was taped with $0.254-\mathrm{mm}(0.01-\mathrm{in}$.) white cotton tape and bonded with resin. The finished core and coil assembly is stwown in Figure A-3. The core is clamped with a steel angle similar to an actual power transformer. 


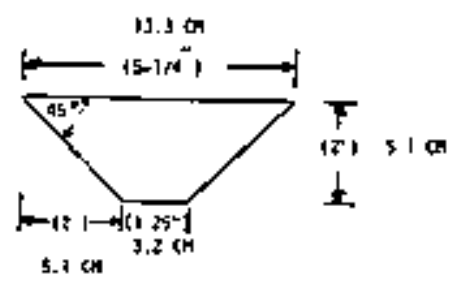

Coet rotke hereing

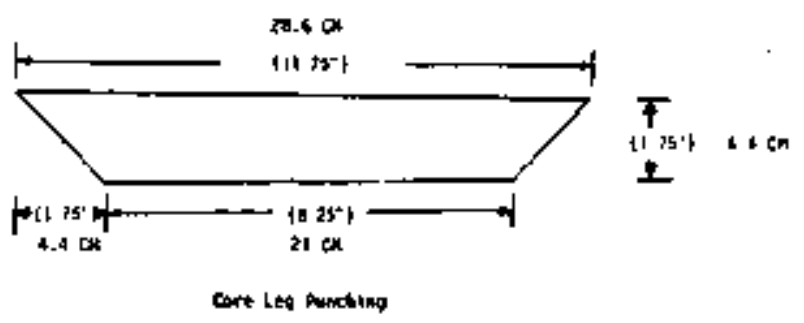

Figure A-1. Core Punching Design
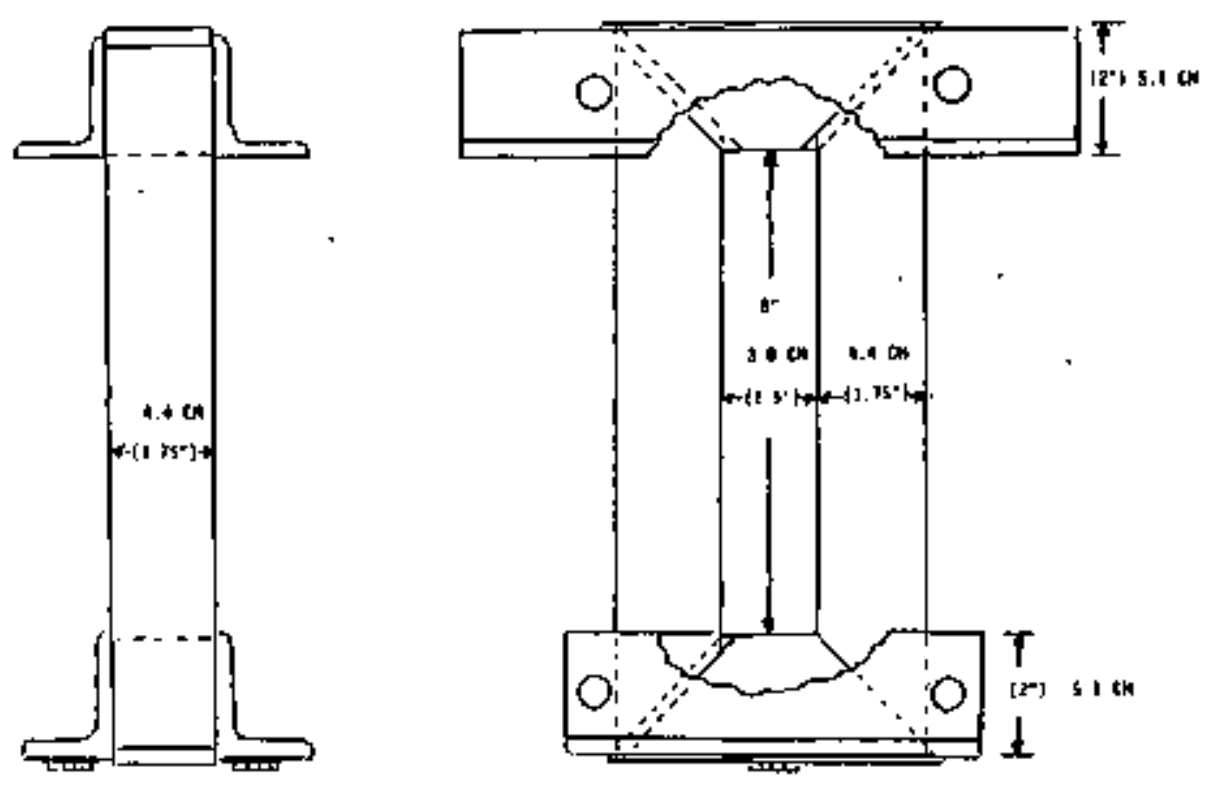

Figure A-2. Core Assembly

A-2 


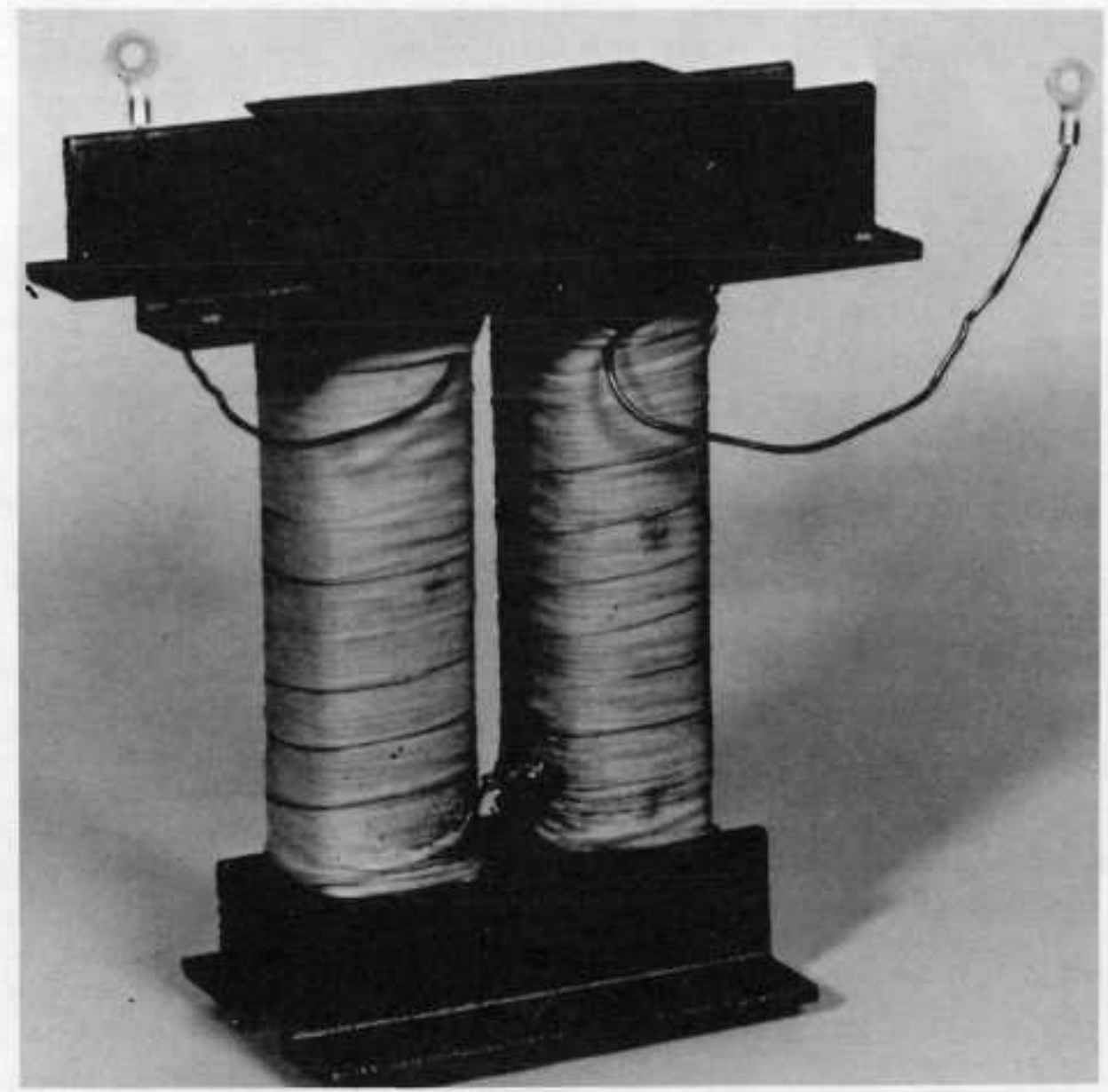

Figure A-3. Finished Core and Coil Assembly 


\section{TANK DESIGN}

The core and coil assembly was fitted into an oil-tight steel tank; Figures $\mathrm{A}-4$ and $\mathrm{A}-5$ show the tank's assembly and design. The overall dimensions of the tank were $381 \mathrm{~mm}$ ( $15 \mathrm{in.}$.) by $381 \mathrm{~mm}$ ( $15 \mathrm{in.}$.) by $330.2 \mathrm{~mm}$ (13 in.), and it was made of $2.29 \mathrm{~mm}(0.09 \mathrm{in.})$ thick steel (Westinghouse PDS \# $10101 \mathrm{BC}$ ). Four half couplings were welded to the top of the tank; two were used as "bosses" for bushings (Westinghouse 25D5334 H01) connected to the transformer winding, and two were used for oil filling and venting. All tank welds were oil tight. The tank flange was made of thick steel (Westinghouse PDS 10110AT) with a machined gasket groove, and the tank was finished with ANSI 170 light gray paint.

\section{HOUSING DESIGN}

The housing surrounding the model transformer tank was necessary to simulate a medium-power transformer. Small transformers inherently generate better high-frequency components than medium-power transformers, and the housing was an attempt to modify the sound emanating from the model transformer to accentuate the $120-\mathrm{Hz}, 240-\mathrm{Hz}$, and $360-\mathrm{Hz}$ frequency components of audible sound. Figure A-6 shows the open end of the sound housing. This end, with the oil-filled model transformer, simulates a segment of a wall surface from an actual power transformer. Figure A-7 shows a top view of the model transformer inside the sound housing. Figures A-8 and A-9 show the elevation and end views, respectively.

The housing was made of $0.019 \mathrm{~m}(0.75-\mathrm{in}$.) plywood, with wood braces and corner fills. Damping blocks made of $25.4 \mathrm{~mm}$ ( 1 in.) thick sponge rubber were attached to the inner surfaces; Figure A-10 shows the array of damping blocks inside the end panels of the housing. The housing was then filled with fiberglass mats (Westinghouse PDS $41424 \mathrm{aX}) 12.7 \mathrm{~mm}$ ( $0.5 \mathrm{in.})$ thick and fiberglass sheets (Westinghouse PDS $41424 \mathrm{AB}) 50.8 \mathrm{~mm}$ (2 in.) thick. This fiberglass filled the housing to within $25.4 \mathrm{~mm}$ ( $1 \mathrm{in.}$ ) of the transf ormer tank. 


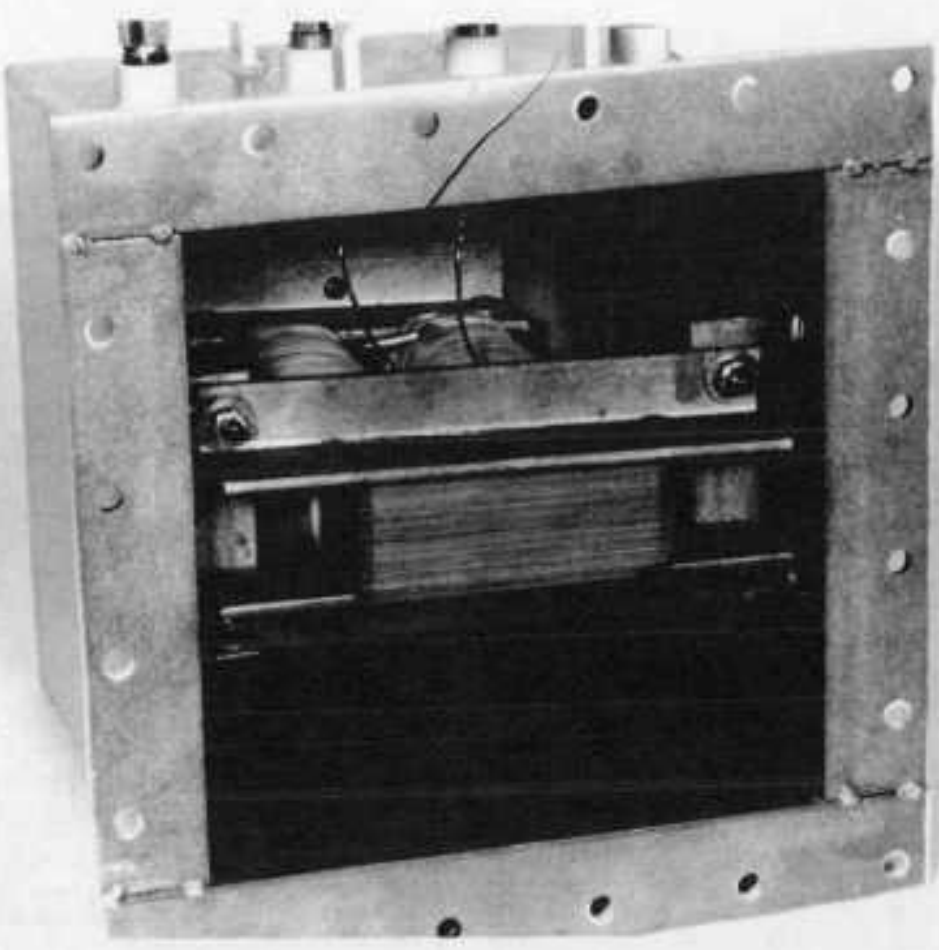

(a)

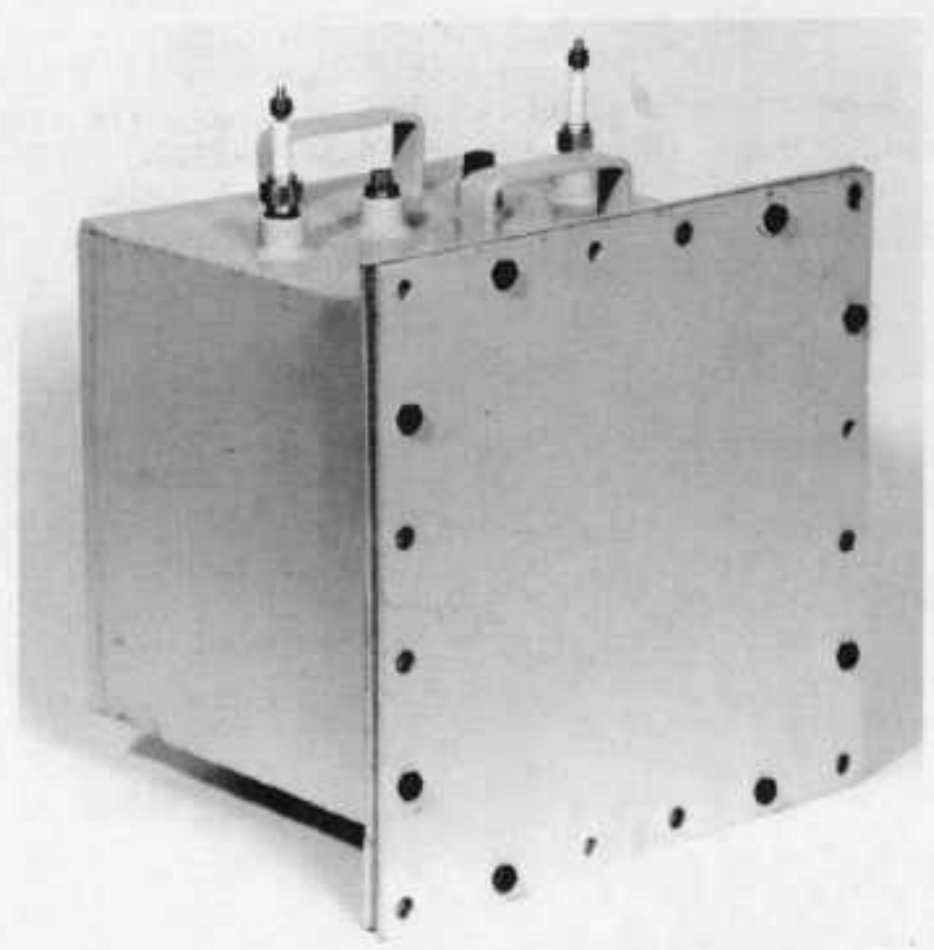

(b)

Figure A-4. (a) Steel Tank I (b) Steel Tank II 

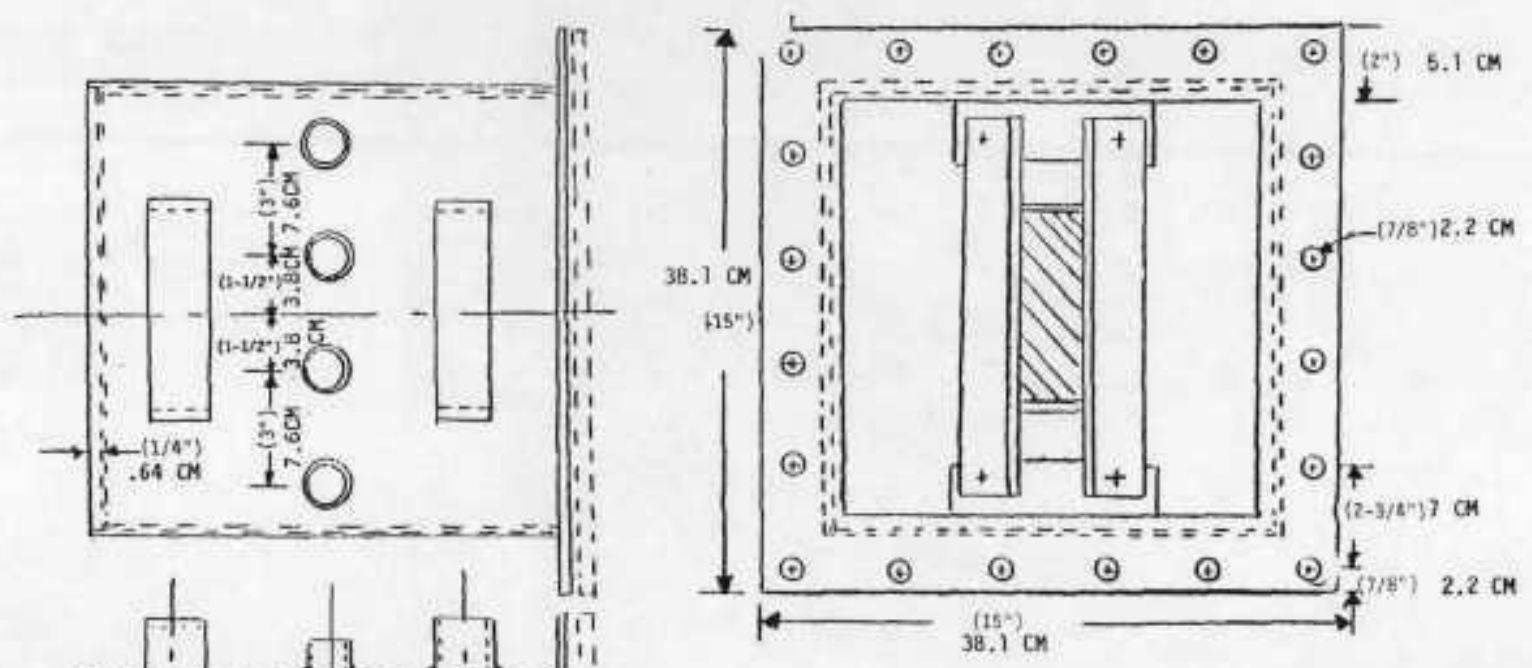

Figure A-5. Tank Assembly D895800-L21 


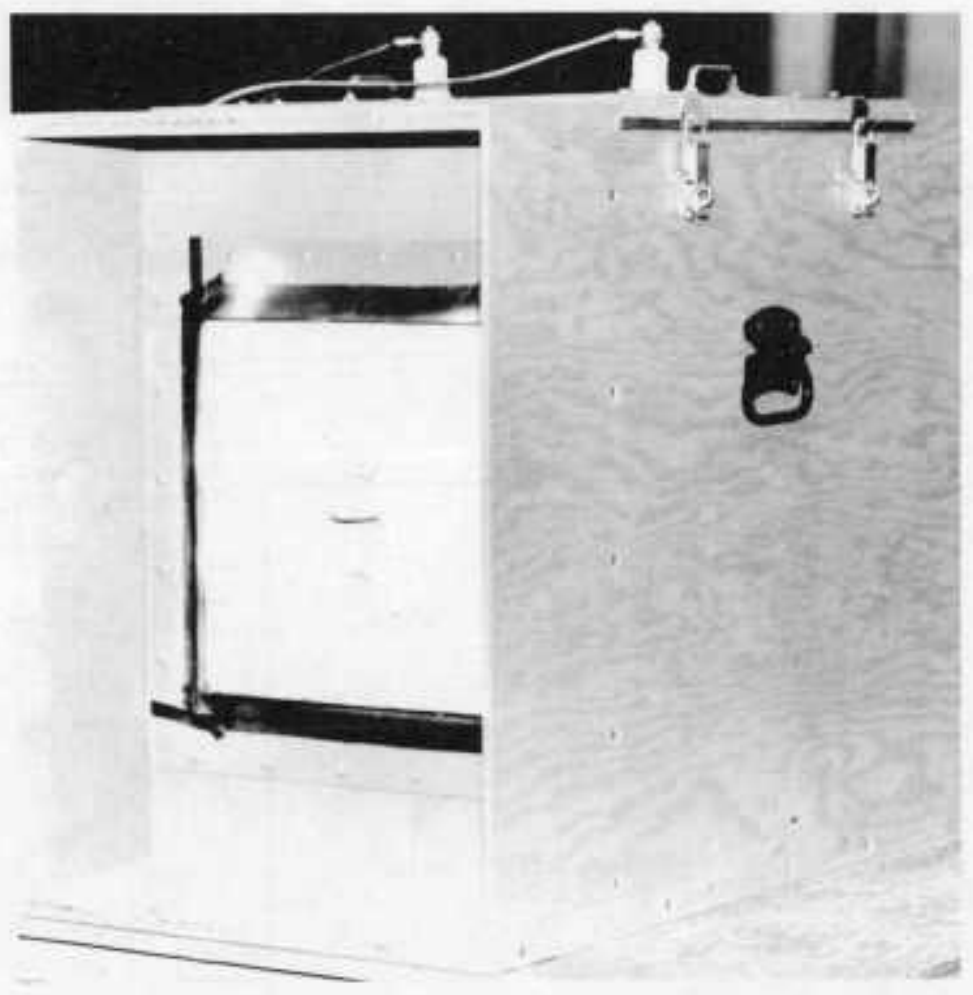

Figure A-6. Housing: Front End

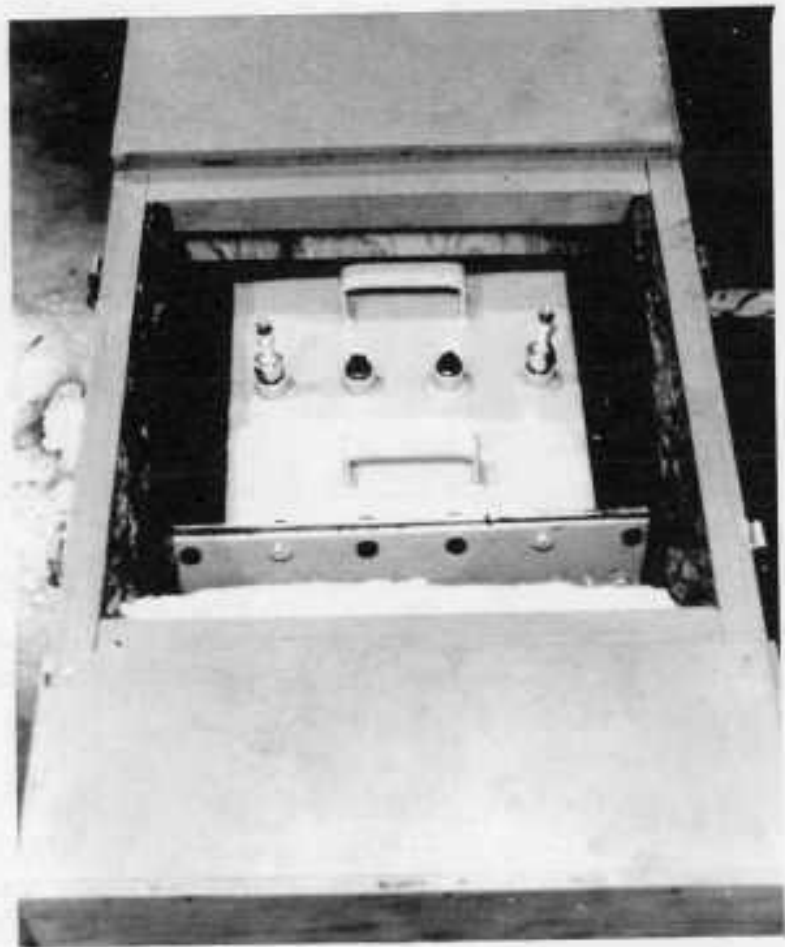

Figure A-7. Housing: Top View

$$
\text { A-7 }
$$




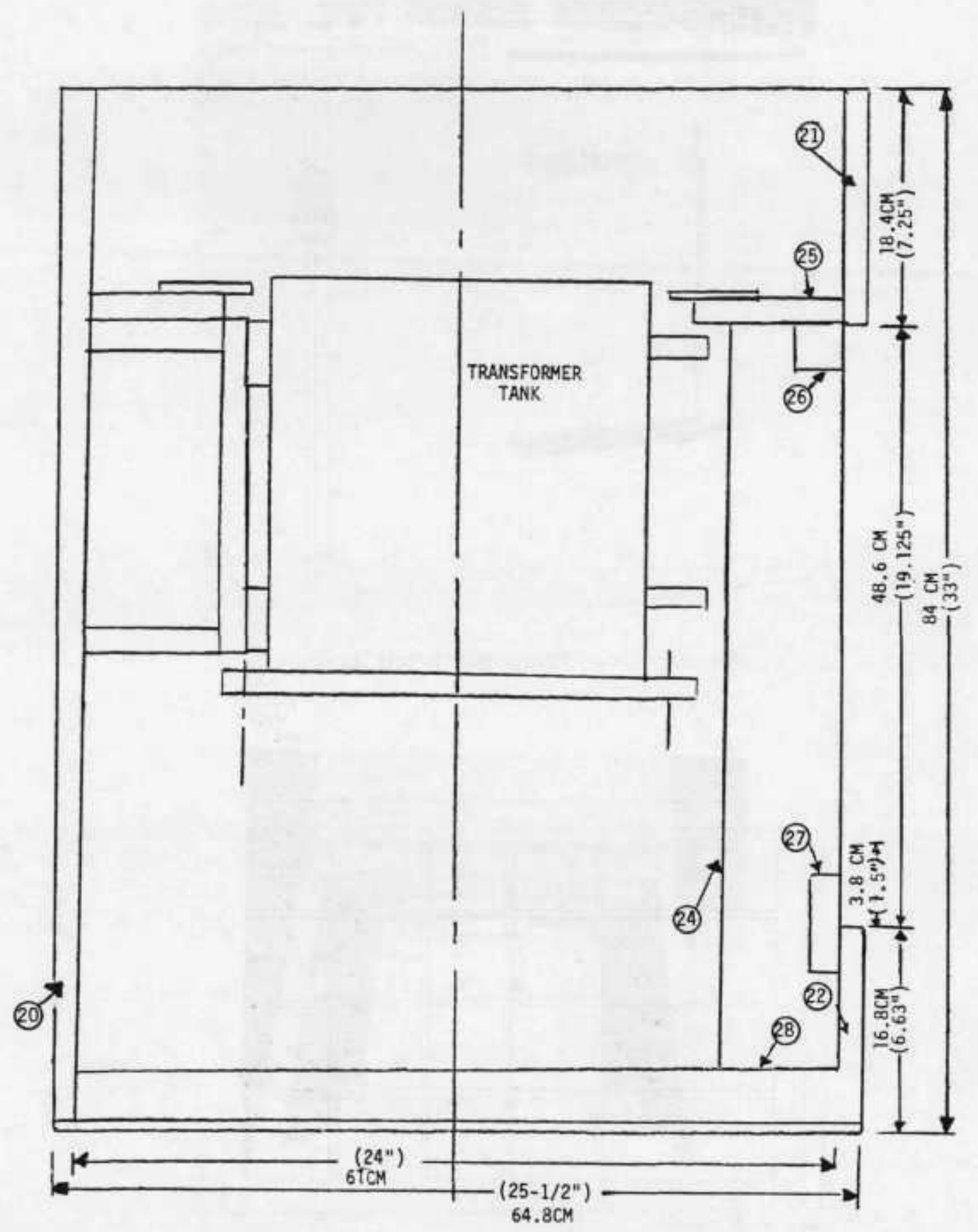

Figure A-8. Elevation View of Sound Housing 


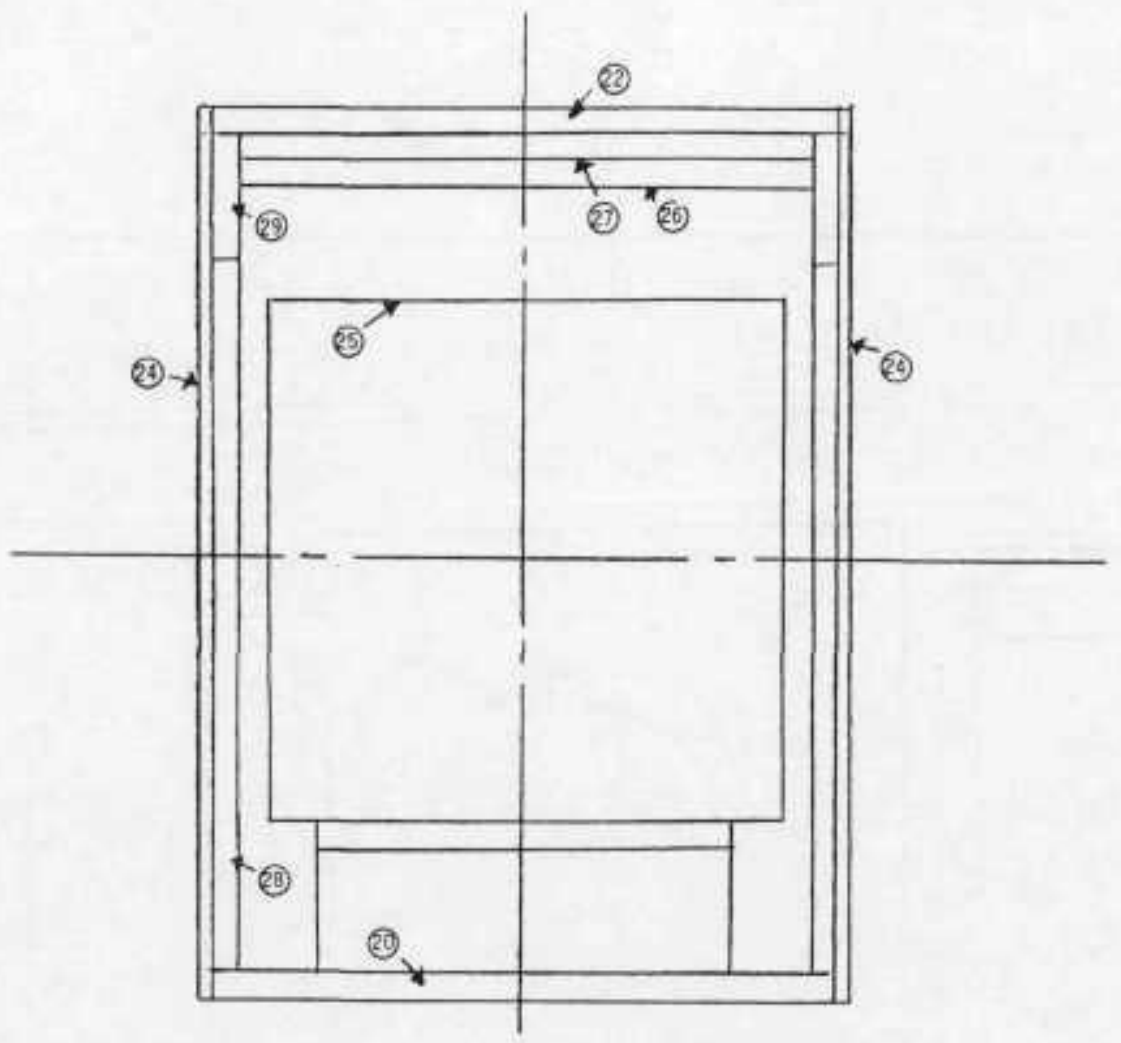

Figure A-9. Side View of Sound Housing

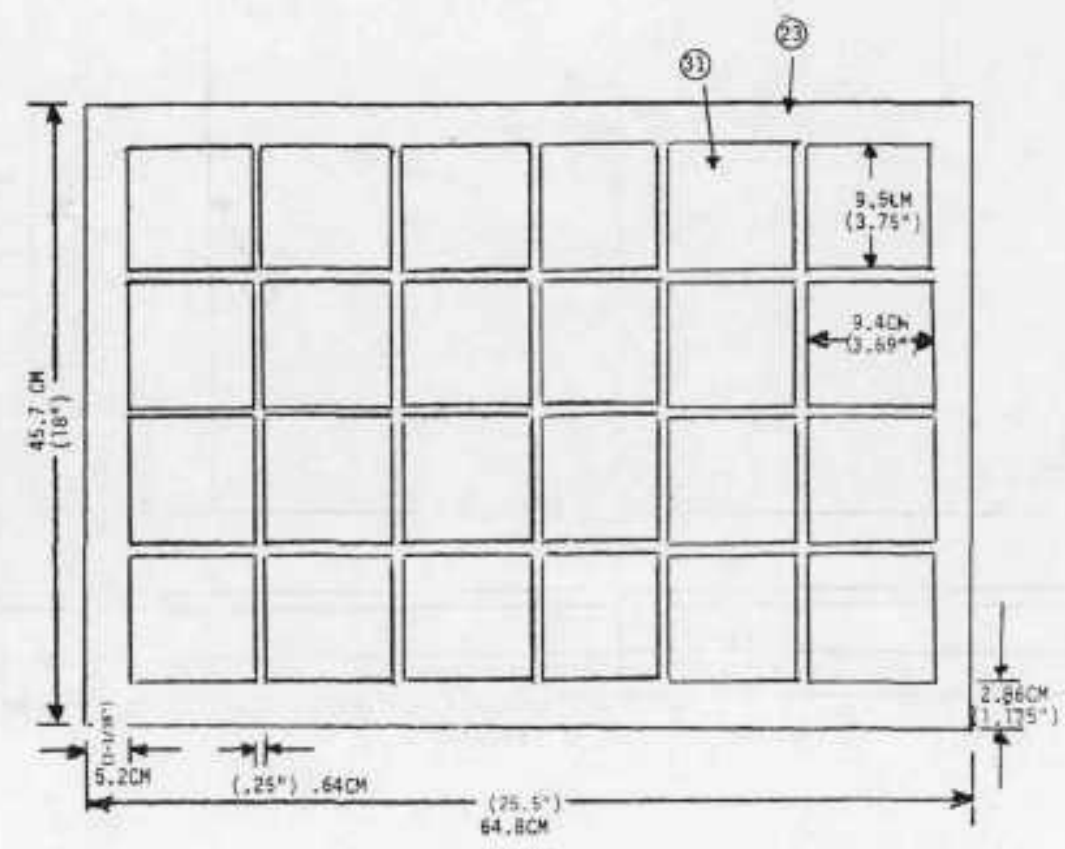

Figure A-10. End Panel With Damping Blocks 


\section{APPENDIX B. DETAILS OF THE ACTIVE NOISE ABATEMENT SYSTEM DESIGN}

The demonstration system (Figures B-1 through B-5) consists of two independent cancellation units and is designed to abate the first three harmonics $(120,240,360 \mathrm{~Hz})$ from a small model transformer. A dynamic microphone in each unit senses the local sound pressure of the transformer. This signal is amplified and conditioned by gain and phase compensation circuits and is applied to a loudspeaker, which radiates a sound pressure wave out of phase with the sound source, resulting in sound cancellation. Each speaker's radiation is simulated by a filter. Using this signal, feedback from the speaker to its own microphone is cancelled by a delay circuit (self-delay), achieving out-of-band stability for the overall cancellation system. The circuitry was designed to achieve $20-\mathrm{dB}$ cancellation at $120 \mathrm{~Hz}, 15-\mathrm{dB}$ cancellation at $240 \mathrm{~Hz}$, and 13-dB cancellation at $360 \mathrm{~Hz}$. Effective sound cancellation is maintained throughout a $20-\mathrm{dB}$ change in transformer output sound level. Each cancellation unit consists of a speaker/microphone assembly, electronic printed-wiring assemblies, and d.c. power supplies.

Speaker assemblies are made up of inexpensive, high-fidelity components. High-compliant $127-\mathrm{mm}$ (5-in.) speakers were mechanically modified by removing the voice coil dust cover and gluing a fiberglass disk in its place. This resulted in additional mass-loading on the voice coil and lowered the mechanical resonance frequency. The speaker was then mounted in a 6555 -cc (400-in. $\left.{ }^{3}\right)$ closed-back enclosure. When installed in the enclosure, the speaker had a mechanical resonance frequency of $50 \mathrm{~Hz}$ and a $Q$ of 1.2. A precision $B \& K$ condenser microphone was mounted by brackets to the side of the enclosure, $102 \mathrm{~mm}$ ( $4 \mathrm{in}$.) from the center of the speaker.

The electronic circuitry (Figure B-6) was fabricated on printed wiring boards. It performed three basic functions: power amplification, selffeedback cancellation, and gain/phase correction. A monolithic 7W power amplifier was used to drive the speaker. The amplifier was mounted directly on the printed circuit board, and plating on the board provided the necessary heat sink for thermal dissipation.

Cancellation of speaker feedback was achieved by generating a signal with the same gain/phase relationship as the speaker and subtracting this signal from the microphone signal. The speaker/microphone transfer function was obtained by experimentally measuring the gain and phase at the microphone output while driving the speaker with a swept frequency. Using a computer, a transfer function was curve-fitted to the measured data (Figure B-7). This transfer function was then simulated, using active filters. The transport delay between the speaker and microphone was simulated by using active all-pass filter sections. Both amplitude and phase of the simulated speaker output were adjustable, to obtain optimum results. Feedback cancellation ranges from $-20 \mathrm{~dB}$ at $125 \mathrm{~Hz}$ to $0 \mathrm{~dB}$ at $3 \mathrm{kHz}$ (Figure B-8). It is anticipated that, with additional effort, the amplitude 


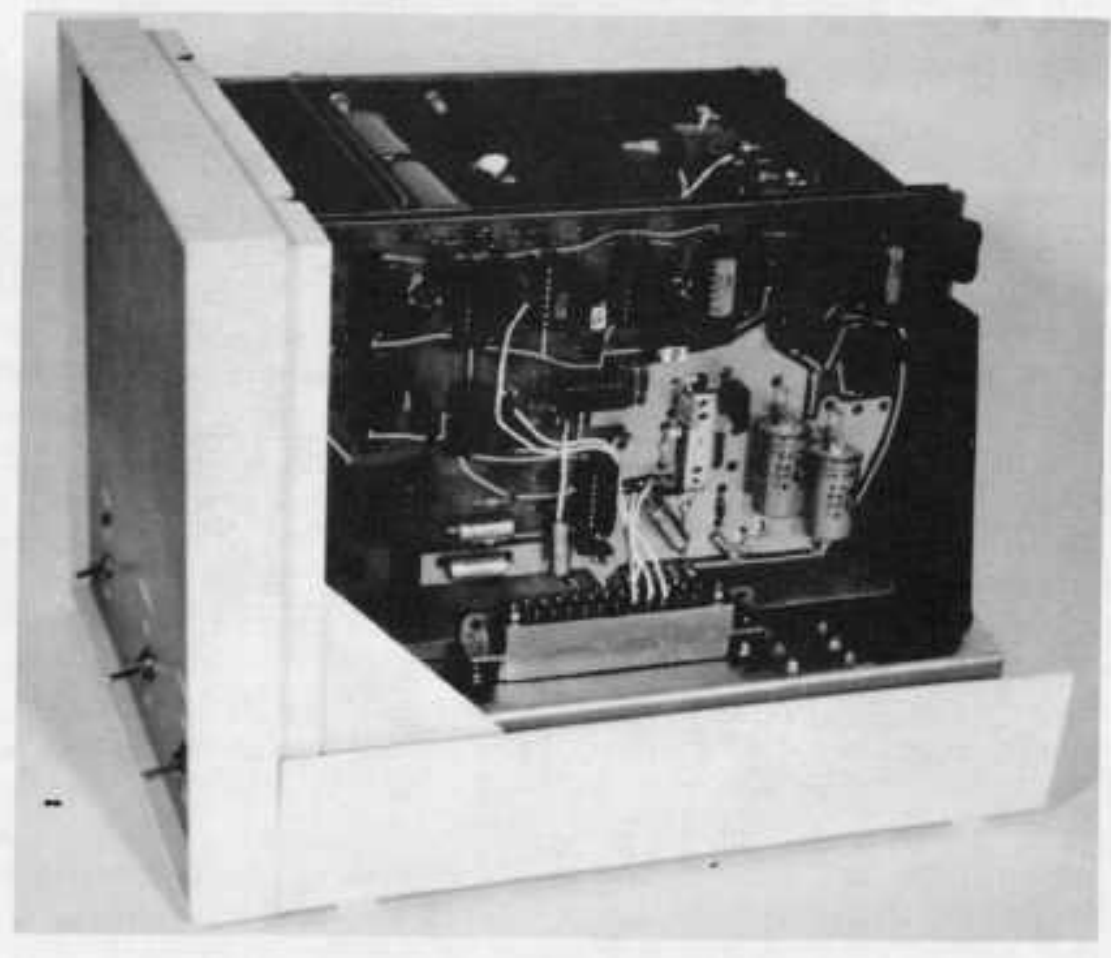

Figure B-1. Demonstration Unit I

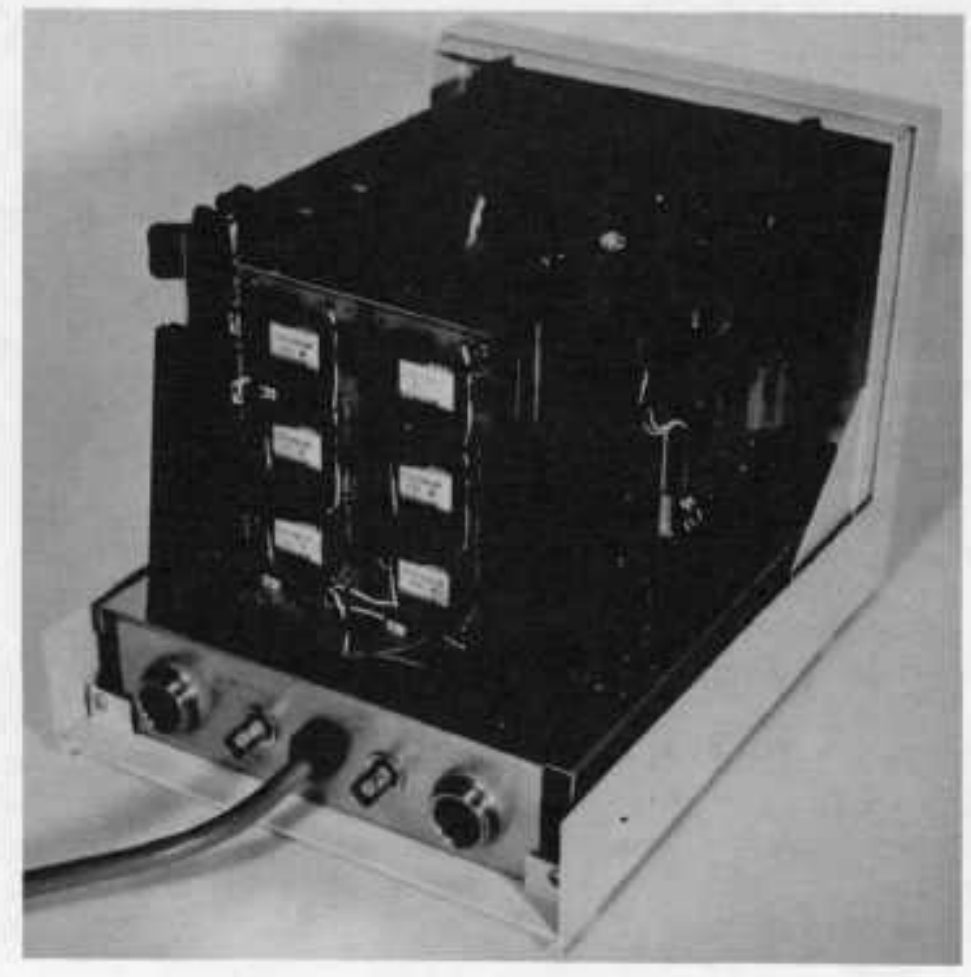

Figure B-2. Demonstration Unit II 


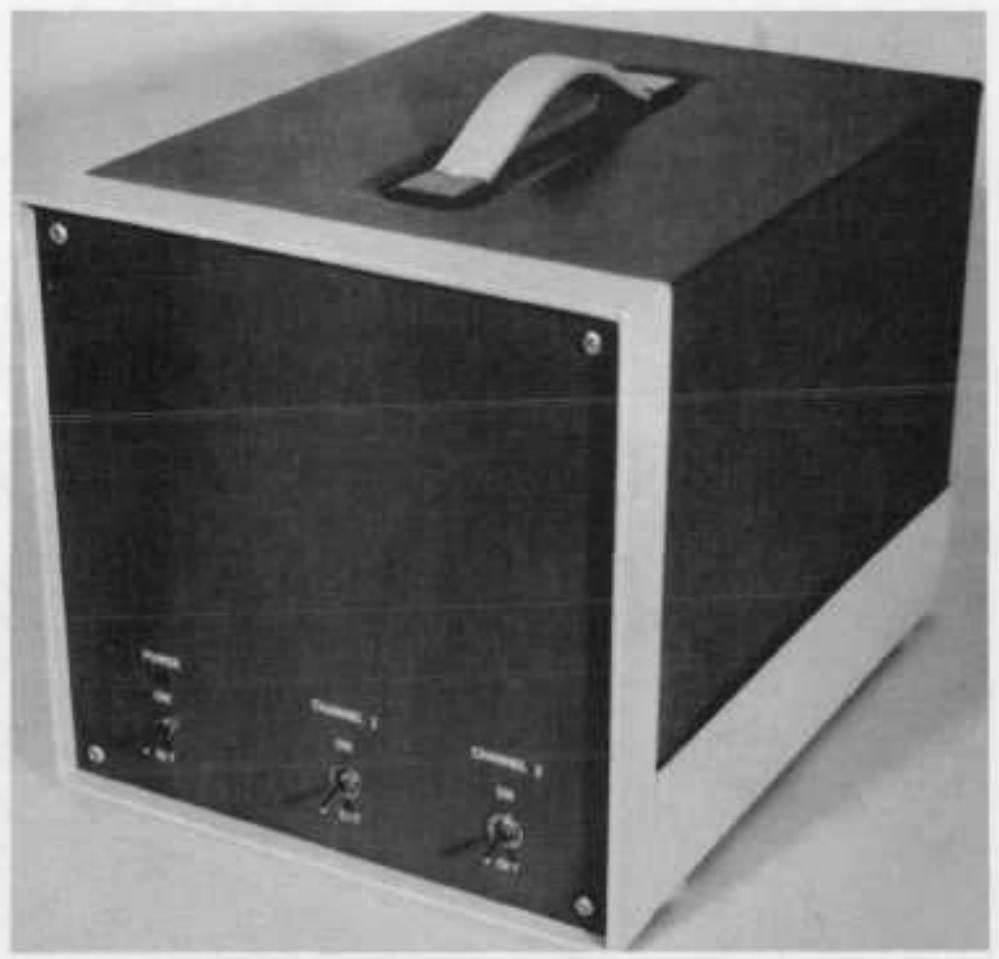

Figure B-3. Demonstration Unit III

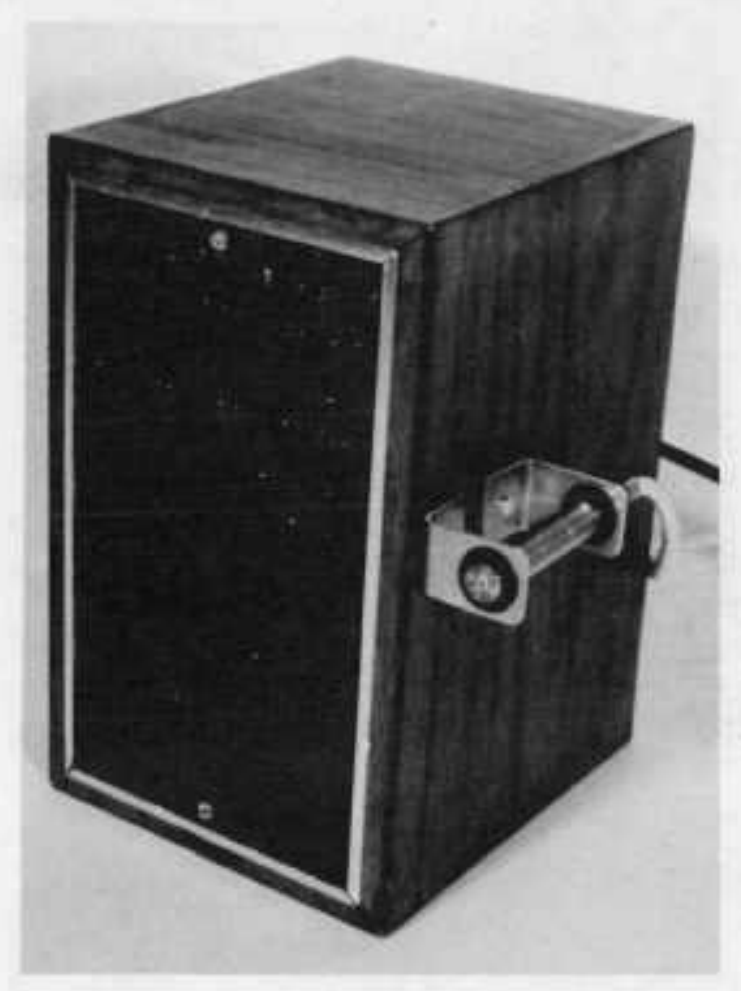

Figure B-4. Demonstration Unit IV

B-3 


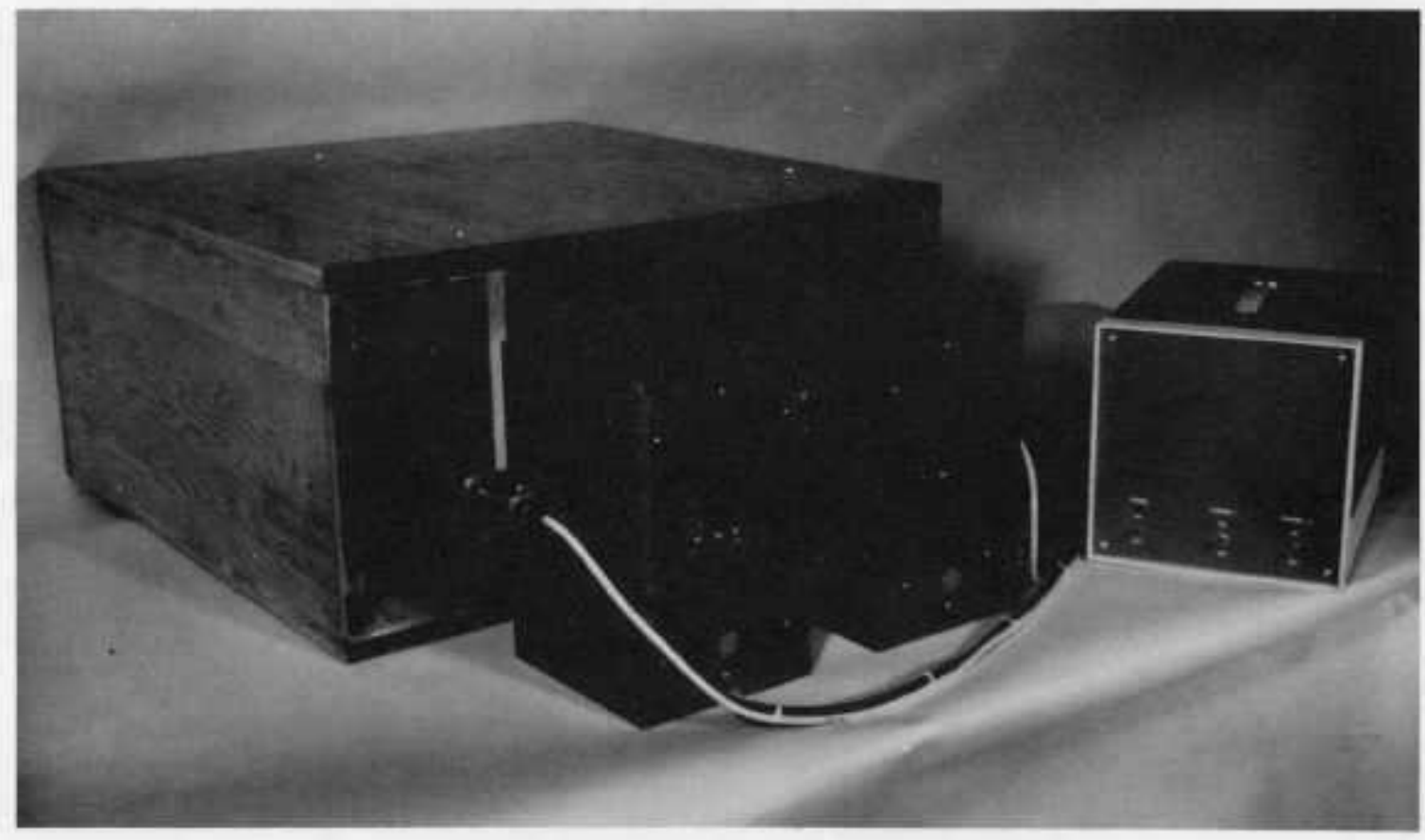

Figure B-5. Demonstration Unit V 


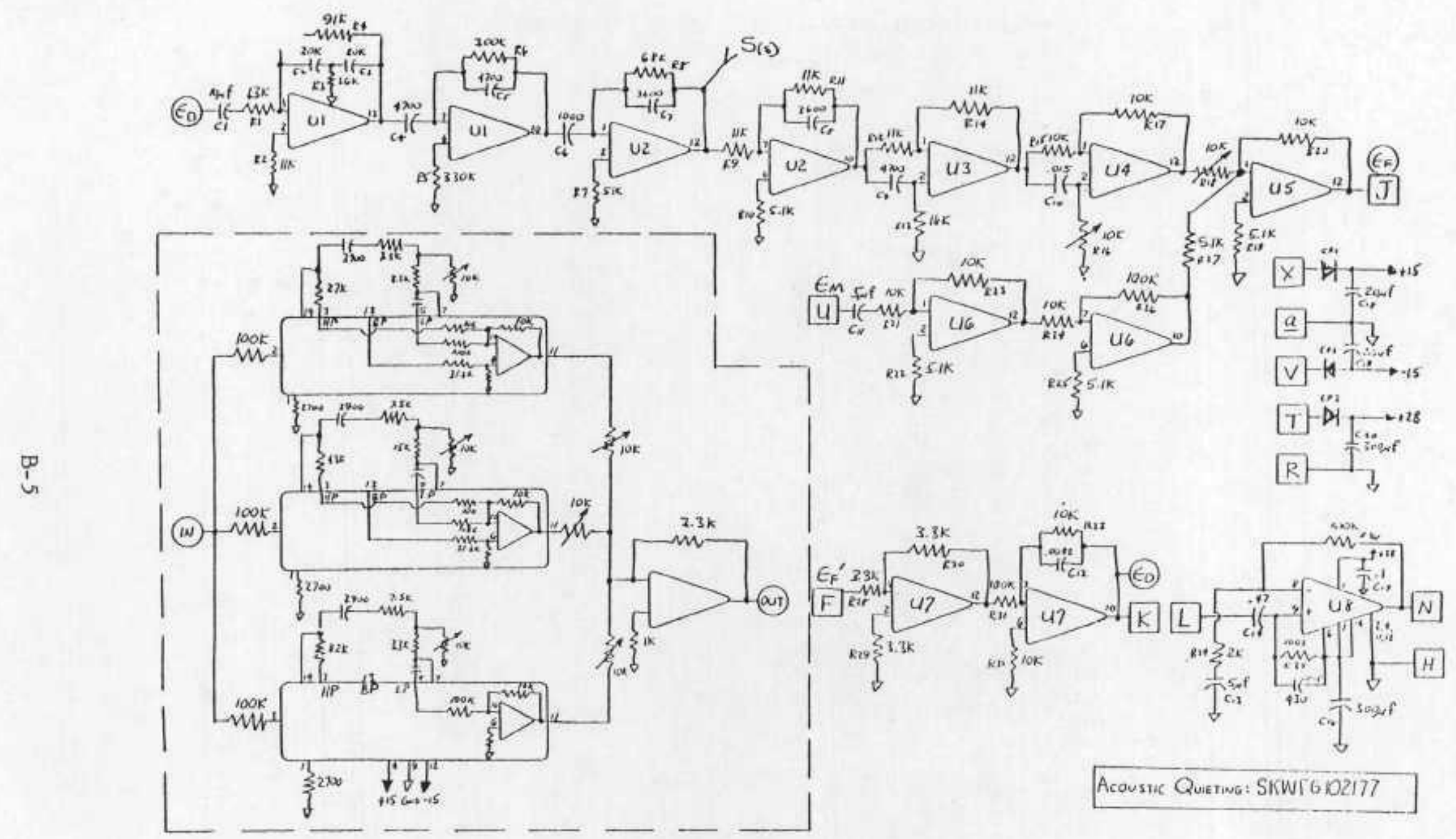

Figure B-6. Circuit Diagram 


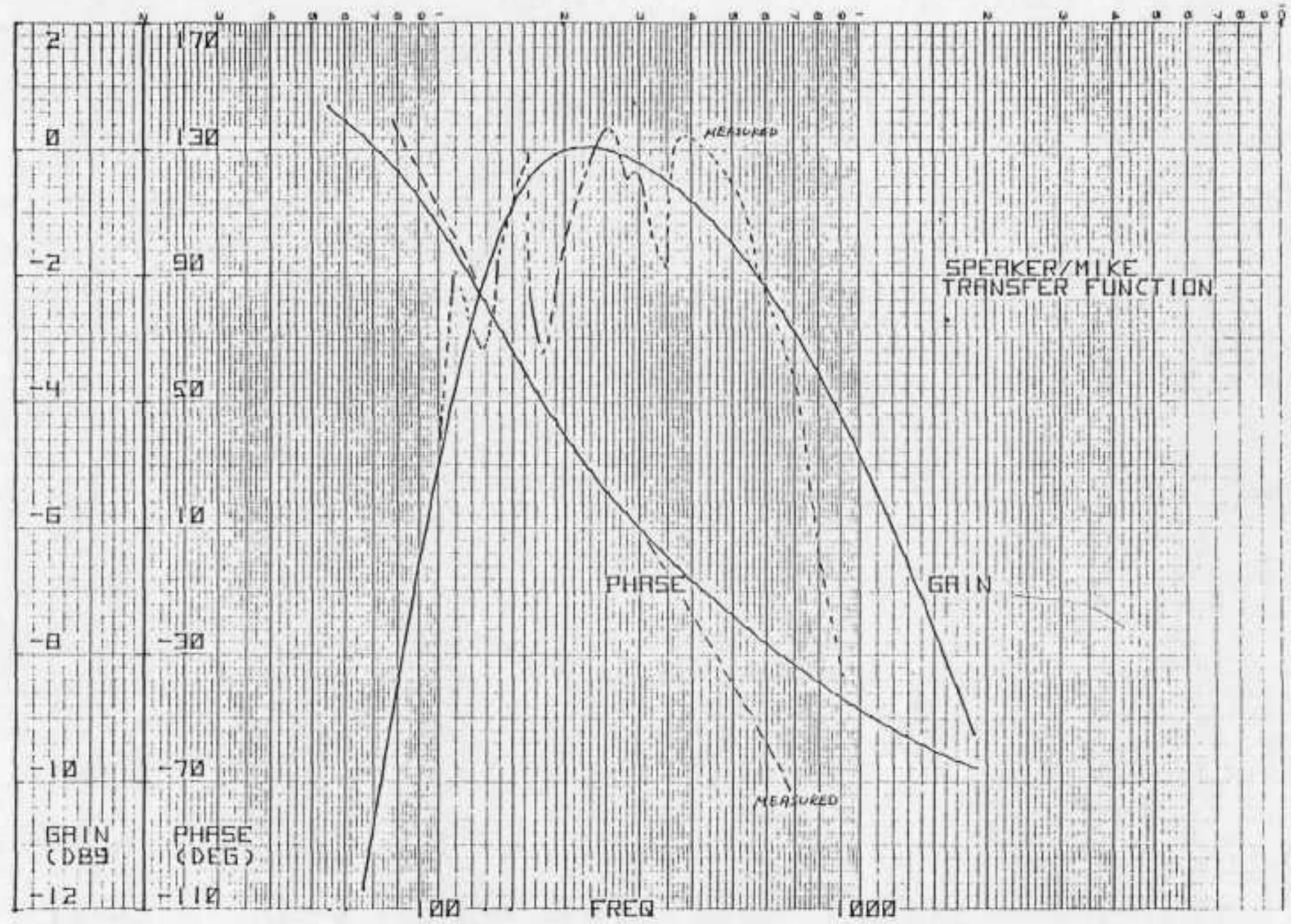

Figure B-7. Speaker/Microphone Transfer Function 


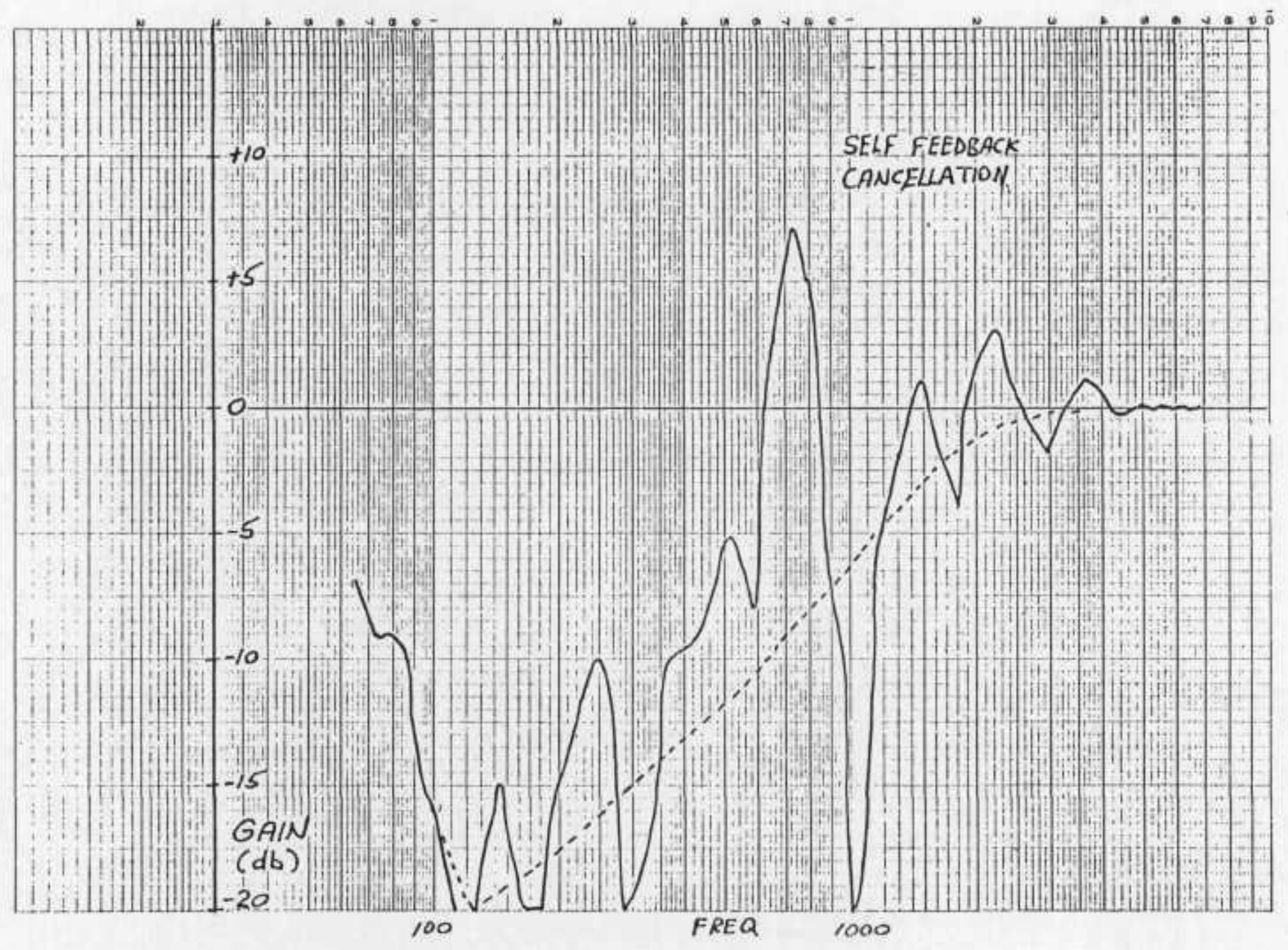

Figure B-8. Feedback Circuit Performance 
and phase adjustments may be predetermined before applying the system to a full-scale transformer.

The remaining signal from the microphone is a true measure of the sound pressure level of the transformer. To obtain maximum noise reduction in the far field of the transformer, additional gain and phase corrections must be implemented. To simplify stability and adjustment considerations, three filters were incorporated to reduce the sound at the three specific frequencies: 120,240 , and $360 \mathrm{~Hz}$. Individual controls were provided to adjust gain and phase for each of the frequencies. The phase of each frequency component can be adjusted by adjusting the resonant frequency slightly; gain can be adjusted by varying the summing amplifier for each frequency. Monolithic active filters were used for each of the three frequencies, configured as second-order bandpass filters with a $Q$ of 10 (Figure B-9).

Stability problems are eased because of the low out-of-band gain of the filters. Both experimental and computer analysis of the entire speakermicrophone/electronics system were performed. From Figure B-10, it was determined that the gain margin is $20 \mathrm{~dB}$ and the phase margin, $70^{\circ}$, indicating relative stability. Figures $\mathrm{B}-11$ and $\mathrm{B}-12$ show the complete assemblies. 


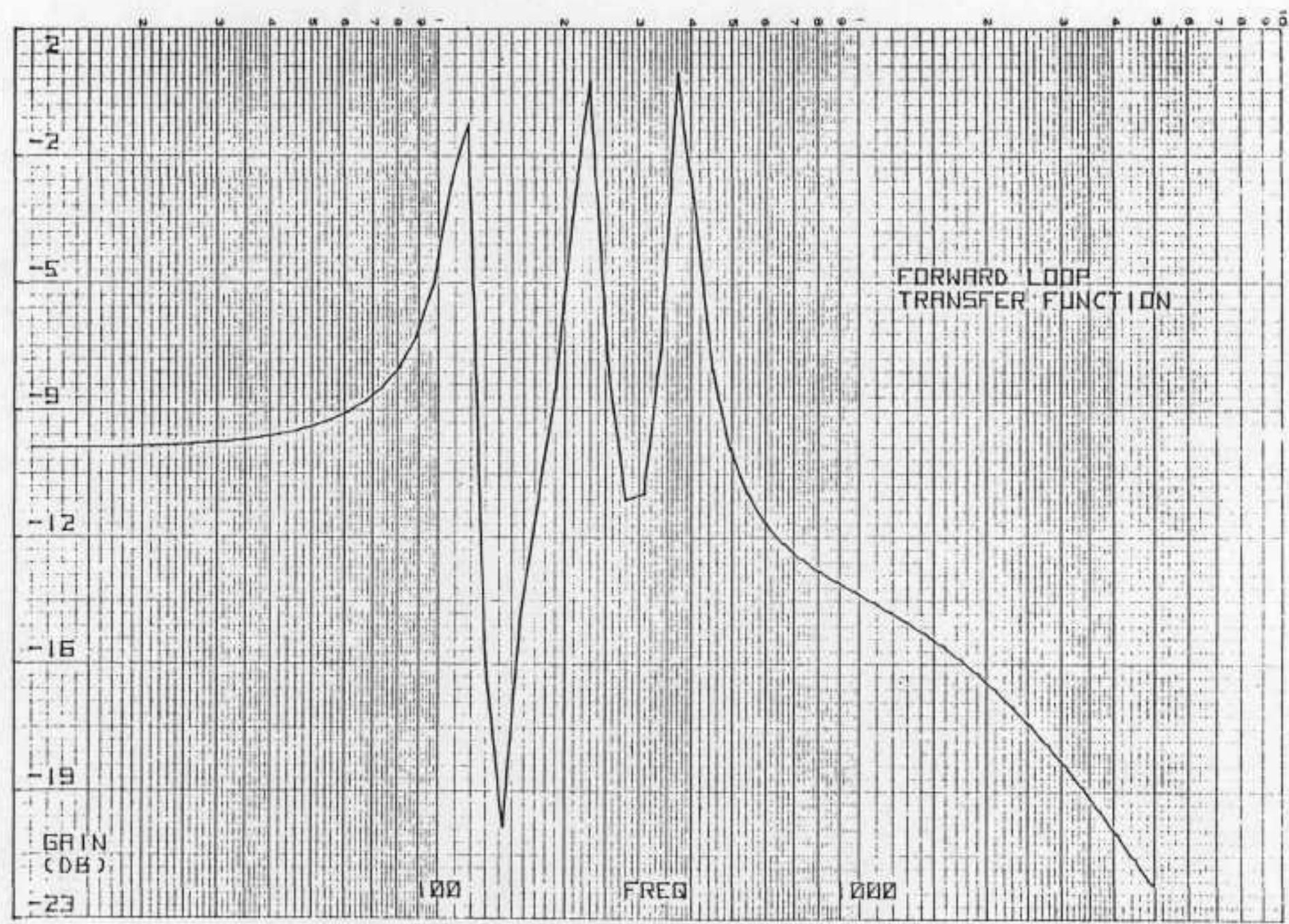

Figure B-9. Narrowband Filter Transfer Function 


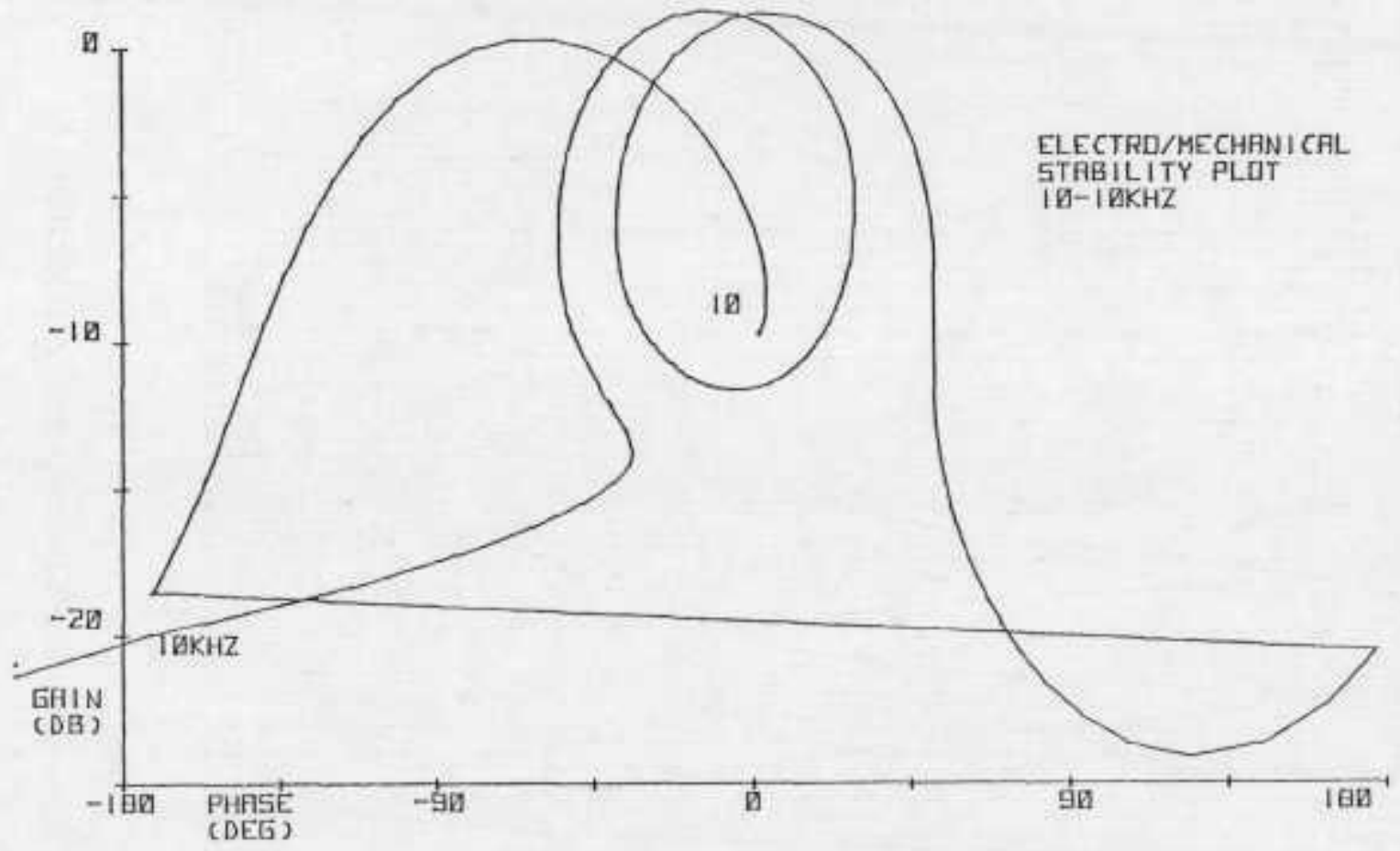

Figure B-10. Stability Plot 


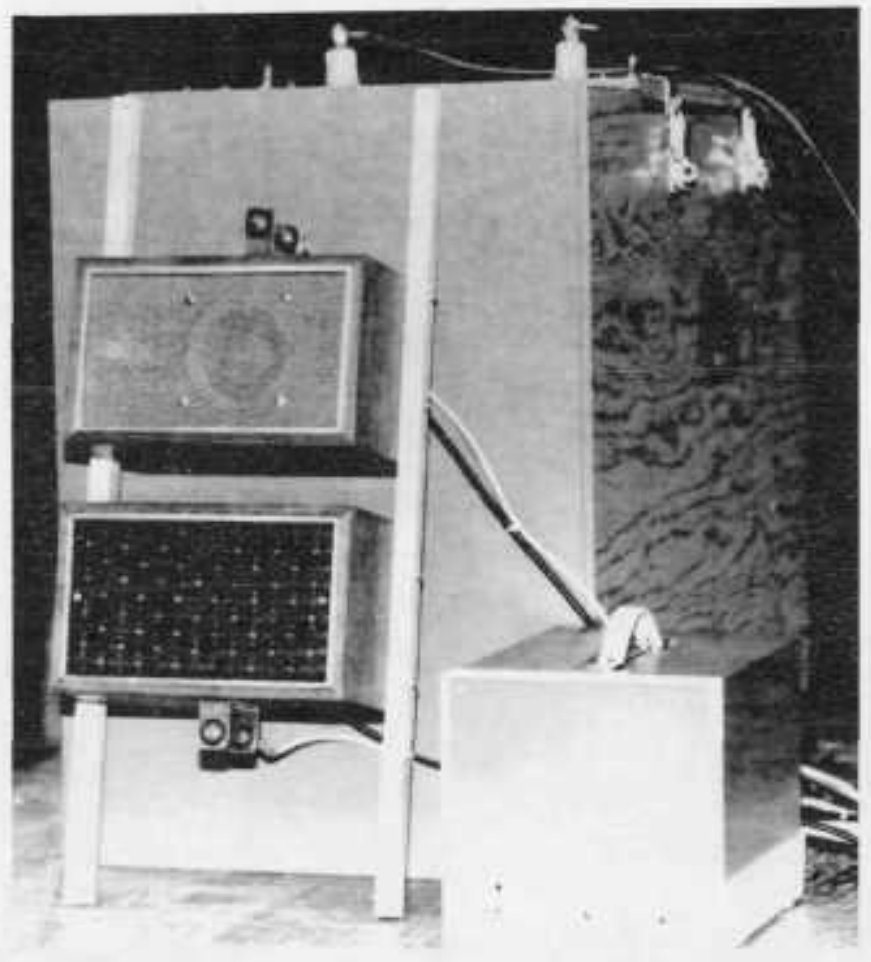

Figure B-11. Completed Assembly I

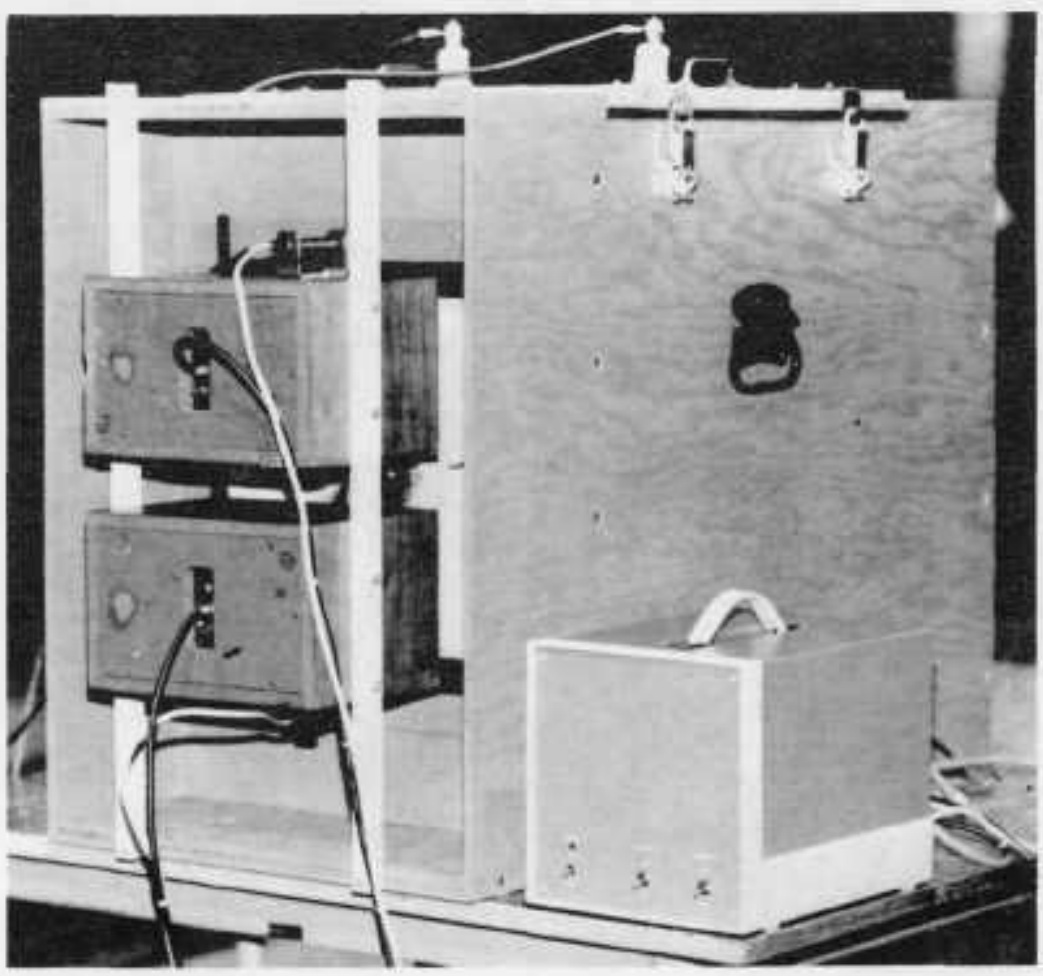

Figure B-12. Completed Assembly II 


\section{APPENDIX C. COMPLETE TEST RESULTS}

\section{RESULTS OF PRELIMINARY TESTING}

Figure C-1 shows the model transformer sound level as the function of frequency, using a $\mathrm{l} 0 \mathrm{a}-\mathrm{Hz}$ bandwidth filter. Curves show the ambient sound ( $R$ line) and the transformer and ambient combined ( $B$ tine). All sound levels are A-weighted. The peak levels at harmonics of the line frequency are summarized below.

Frequency ( $\mathrm{Hz})$

120

240

360

480

\section{Amsient $(d B(A))$}

37

20

20

20
Transformer (dB(A))

44

54

49

46

At 240,360 , and $480 \mathrm{~Hz}$, the transformer is sufficiently above the ambient, so that the readings can be attributed to the transformer. At $120 \mathrm{~Hz}$, however, the transformer is only $7 \mathrm{~dB}$ above the ambient. Hence, the $120-\mathrm{Hz}$ component due to the transformer alone would be at about $43 \mathrm{~dB}(\mathrm{~A})$.

\section{RESULTS OF VARIATHON OF APPLIED VOLTAGE}

Figure C-2 shows the transformer sound levels as a function of frequency for four different voltages applied to the transformer: 100, 110, 120 , and $130 \mathrm{~V}, 60 \mathrm{~Hz}$, single phase. The resul ts are summarized below.

\begin{tabular}{cccccc} 
Excitation & \multicolumn{4}{l}{ Sound Level $(\mathrm{dB}(\mathrm{A}))$} & $10-\mathrm{Hz}$ Bandwidth \\
Voltage $(V)$ & & $120 \mathrm{~Hz}$ & $\underline{240 \mathrm{~Hz}}$ & $360 \mathrm{~Hz}$ & $480 \mathrm{~Hz}$ \\
\cline { 2 - 6 } & & & & 35 & 42 \\
100 & 41.5 & 44 & 43 & 47 \\
110 & 42.5 & 46.5 & 43 & 42.5 \\
120 & 41 & 50.5 & 47 & 48.5 & 42 \\
130 & 39 & 53 & 48.5
\end{tabular}

The $120-\mathrm{Hz}$ components were low at all voltages and would not be a factor in choosing the applied voltage. At $130 \mathrm{~V}$, the $240-$ and $360-\mathrm{Hz}$ components are increased but the $480-\mathrm{Hz}$ component is about the same as at lower vol tages. 


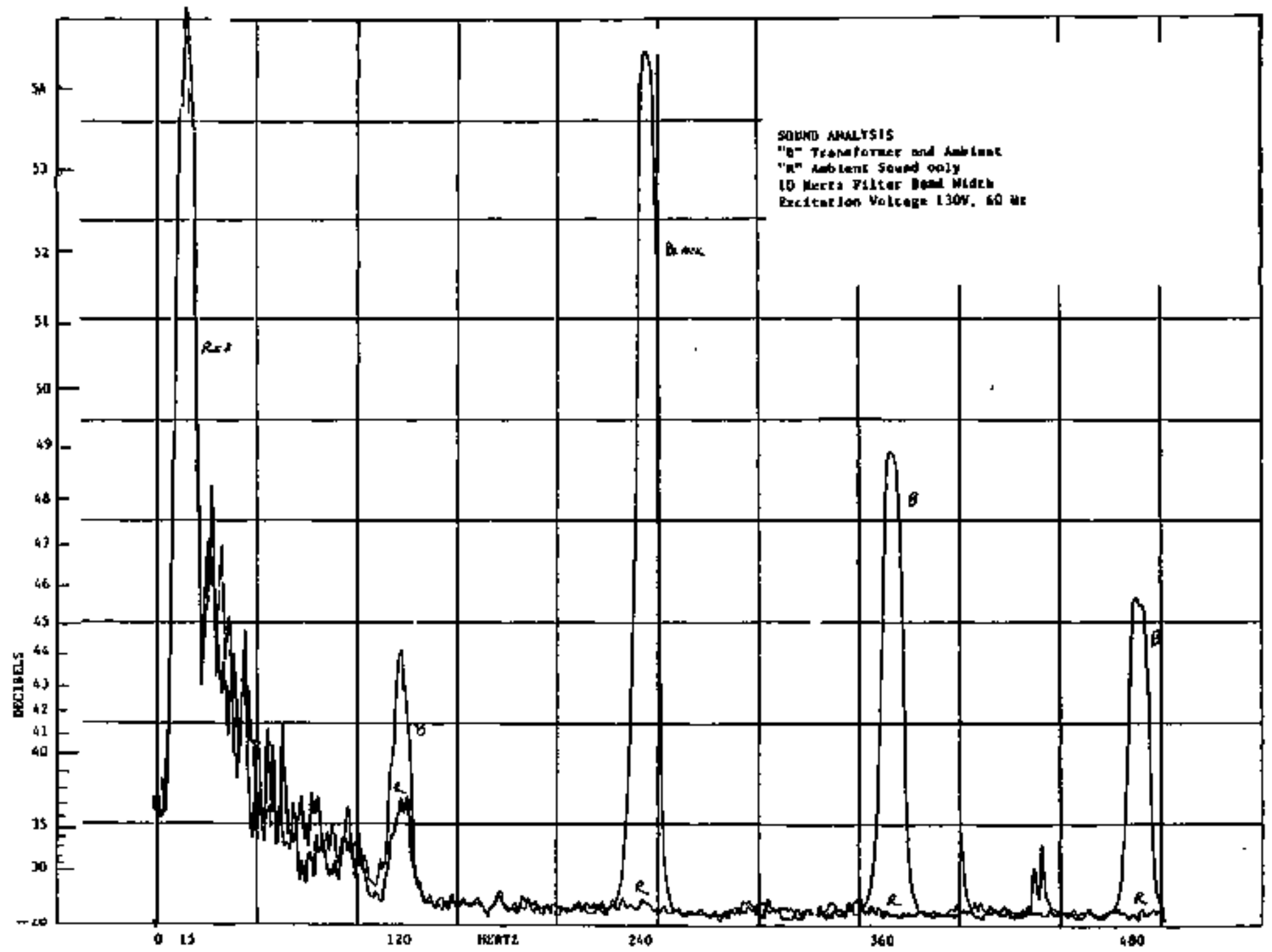

Figure C-1. Transformer Sound Spectrum 


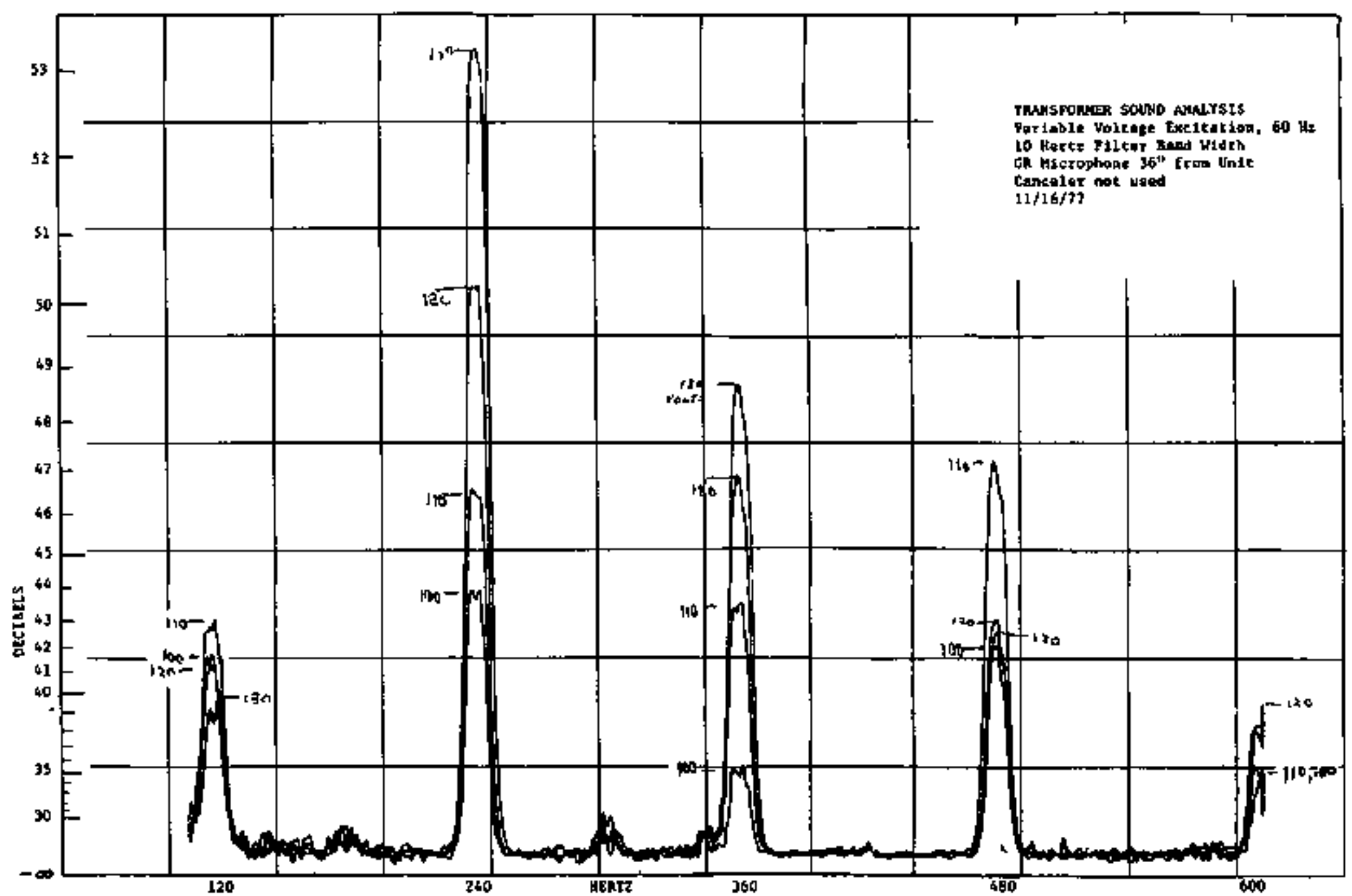

Figure C-2. Transformer Sound Analysis 
Figure C-3 shows the transformer sound level at $120 \mathrm{~V}$, with (C-trace) and without ( $T$-trace) the active noise abatement system. The sound levels at the line frequency harmonics are summarized below.

\begin{tabular}{|c|c|c|c|}
\hline \multirow[b]{2}{*}{$\begin{array}{l}\text { Frequency } \\
(\mathrm{Hz})\end{array}$} & \multicolumn{3}{|c|}{ Sound Level $(\mathrm{dB}(A))$} \\
\hline & $\begin{array}{l}\text { No } \\
\text { Abatement } \\
. \quad \text { (T) } \\
\end{array}$ & $\begin{array}{l}\text { With } \\
\text { Abatement } \\
\text { (C) }\end{array}$ & $\begin{array}{l}\text { Net Sound } \\
\text { Reduction }\end{array}$ \\
\hline $\begin{array}{l}120 \\
240 \\
360 \\
480\end{array}$ & $\begin{array}{l}39 \\
50 \\
39 \\
35\end{array}$ & $\begin{array}{l}18 \\
13 \\
18 \\
35\end{array}$ & $\begin{array}{r}21 \\
37 \\
19 \\
0\end{array}$ \\
\hline
\end{tabular}

The figure shows that, at $180 \mathrm{~Hz}$, a slight increase in sound level is observed with the noise abatement system.

Figure C-4 shows the sound levels at an exçitation voltage of $130 \mathrm{~V}$. The values are listed below.

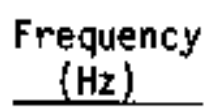

120

240

360

480

\begin{tabular}{|c|c|c|}
\hline \multicolumn{3}{|c|}{ Sound Level ( $\mathrm{B} B(\mathrm{~A}))$} \\
\hline $\begin{array}{c}\text { No } \\
\text { Abatement } \\
\text { (T) }\end{array}$ & $\begin{array}{l}\text { With } \\
\text { Abatement } \\
\text { (c) }\end{array}$ & $\begin{array}{l}\text { Het Sound } \\
\text { Reduction }\end{array}$ \\
\hline
\end{tabular}

37

53

43

21
17

28

30

2I
20

25

13

Again, the figure shows an increase in the $180-\mathrm{Hz}$ component with the abatement system in use.

These tests demonstrate the feedback teature of the cancellation system; as the excitation voltage is varied, sound abatement still takes place.

\section{RESULTS OF SOUND DIRECTIVITY TESTS}

Sound level tests were made on a $3.4 \mathrm{~m}(11 \mathrm{ft})$ diameter circle about the unit (Section IV, Figure 3). The measured sound levels are tabulated in Table C-I. Station 1 corresponds to the open face of the transformer housing, where the two sound cancellation units were attached. 


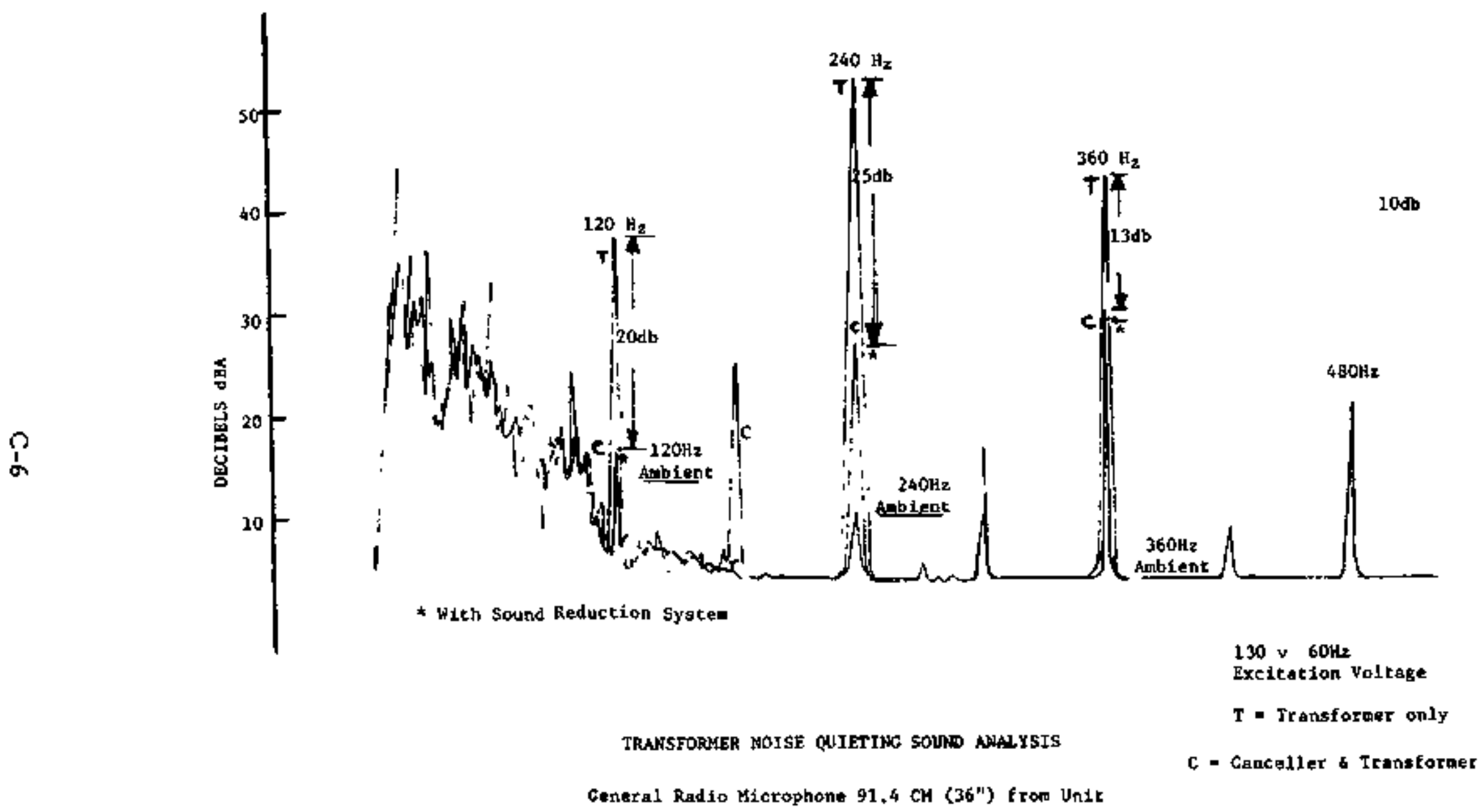

Figure C-4. Transformer Noise Abatement Analysis 


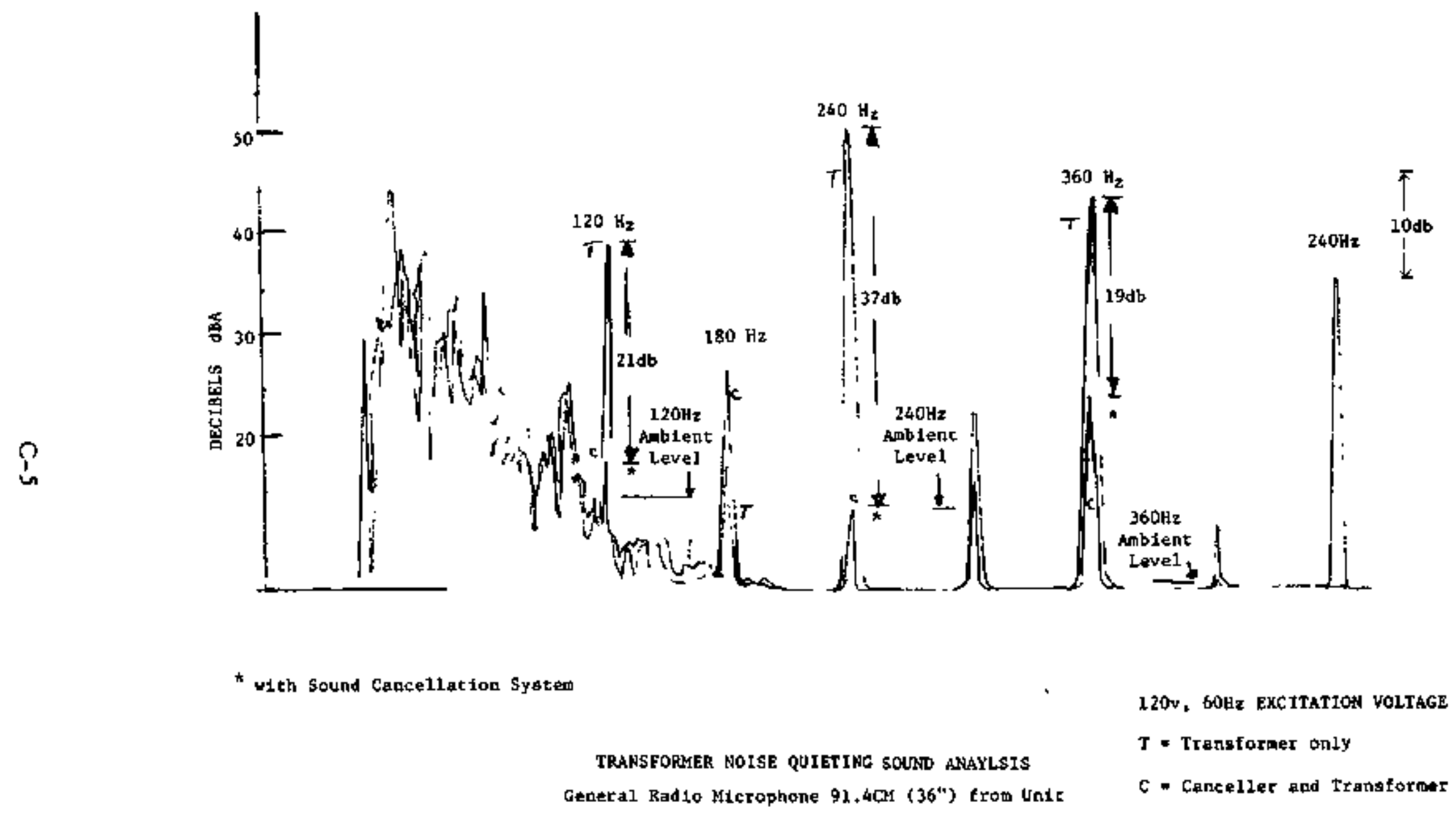

Figure C-3. Transformer Noise Abatement Analysis 
Table C-1. Sound Level Directivity Tests of Model Transformer at Rated Voltage (120V)

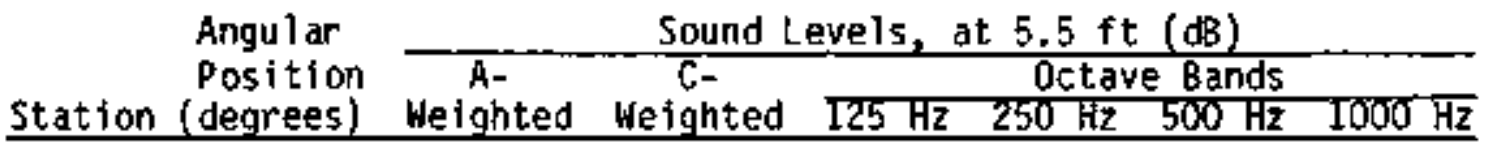

Without Active Noise Abatement System

$\begin{array}{lrllllll}1 & 0 & \mathbf{4 5 . 5} & \mathbf{5 5 . 9} & 38.5 & 52.2 & 44.6 & 36.5 \\ 2 & 45 & 42.7 & 54.4 & 39.3 & 46.8 & 41.0 & 33.4 \\ 3 & 90 & 39.5 & 53.8 & 39.0 & 46.1 & 35.2 & 29.9 \\ 4 & 135 & 37.9 & 53.4 & 41.9 & 43.2 & 35.0 & 28.5 \\ 5 & 180 & 39.2 & 54.0 & 42.0 & 42.3 & 38.5 & 31.0 \\ 6 & 225 & 37.8 & 55.0 & 42.8 & 39.5 & 36.1 & 31.4 \\ 7 & 270 & 41.8 & 55.8 & 41.0 & 46.7 & 37.9 & 35.4 \\ 8 & 315 & 44.3 & 55.6 & 41.9 & 40.7 & 42.3 & 34.8\end{array}$

With Active Noise Abatement System

$\begin{array}{rrrrrrrr}1 & 0 & 42.9 & 54.0 & 37.5 & 35.9 & 41.7 & 37.9 \\ 2 & 45 & 41.1 & 53.4 & 37.7 & 37.9 & 41.0 & 33.8 \\ 3 & 90 & 36.1 & 52.2 & 37.8 & 36.3 & 33.4 & 30.8 \\ 4 & 135 & 37.9 & 53.5 & 41.2 & 43.8 & 33.3 & 29.0 \\ 5 & 180 & 41.3 & 54.5 & 42.1 & 45.9 & 37.3 & 31.5 \\ 6 & 225 & 38.7 & 55.1 & 42.4 & 41.5 & 37.4 & 29.9 \\ 7 & 270 & 39.3 & 55.6 & 41.8 & 44.0 & 35.7 & 32.6 \\ 8 & 315 & 40.9 & 54.9 & 39.5 & 41.0 & 32.8 & 36.7\end{array}$

Actual Abatement

$\begin{array}{rrrrrrrr}1 & 0 & 2.60 & 1.90 & 1.00 & 16.30 & 2.90 & -1.40 \\ 2 & 45 & 1.60 & 1.00 & 1.60 & 8.90 & 0.00 & -0.40 \\ 3 & 90 & 3.40 & 1.60 & 1.20 & 9.80 & 1.80 & -0.90 \\ 4 & 135 & 0.00 & -0.10 & 0.70 & -0.60 & 1.70 & -0.50 \\ 5 & 180 & -2.10 & -0.50 & -0.10 & -3.60 & 1.20 & -0.50 \\ 6 & 225 & -0.90 & -0.10 & 0.40 & -2.00 & -1.30 & 1.50 \\ 7 & 270 & 2.50 & 0.20 & -0.30 & 2.70 & 2.20 & 2.00 \\ 8 & 315 & 3.40 & 0.70 & 2.40 & 8.70 & 9.50 & -1.90\end{array}$




\section{APPENDIX D. PSYCHOACOUSTIC DEMONSTRATION EXPERIMENT ON TRANSFORMER NOISE REDUCTION}

On January 5, 1978, an active sound cancellation system for transformer noise was demonstrated at the Westinghouse Transformer Division in Shaton, Pennsylvania. About 16 scientists and engineers attended the demonstration, which was held in a large hemi-anechoic room. The model transformer was located on a table, with the group of observers standing about 3 to $4 \mathrm{~m}$ in front of the device. The transformer had been equipped with an active sound cancellation system that reduced the $120-, 240-$, and $360-\mathrm{Hz}$ pure-tone components of the acoustic noise generated by the transformer. A brief psychoacoustic demonstration experiment was conducted with this group of observers to determine the relative reduction in annoyance due to the transformer noise that could be achieved by the sound cancellation system.

Each member of the group of observers was given a small sheet of paper with four vertical 0- to 10-point annoyance scales (about $7.5 \mathrm{~cm}$ in length) with marks and numerals at the 0,5 , and 10 points. The group listened to the quiet ambient background (about $36 \mathrm{~dB}(\mathrm{~A})$ ) in the hemianechoic room for about $15 \mathrm{~s}$; this was assigned an annoyance value of 0 . The group then listened for about $15 \mathrm{~s}$ to the workshop noise that penetrated the room (about $75 \mathrm{~dB}(A)$ ) when one wall was opened to the factory; this was assigned an annoyance value of 10 . Next the group listened to the noise emitted by the model transformer under four different operating conditions: (J) energized at $120 \mathrm{~V}$ without cancellation, (2) $120 \mathrm{~V}$ with cancellation, (3) $130 \mathrm{~V}$ without cancellation, (4) $130 \mathrm{~V}$ with cancellation. Each condition was heard for 15 to $20 \mathrm{~s}$, and members of the group rated their annoyance on the 0 to 10 scale after each exposure.

The results of the experiment are shown in Figure D-1. The mean relative annoyance scores for the 16 observers were computed for each condition. These values are plotted along the ordinate. The relative sound pressure levels of the $120-, 240-$, and $360-\mathrm{Hz}$ tonal components were measured with a narrowband frequency analyzer. The arithmetic average of the relative sound pressure Jevels of all three components was used to characterize the sound emitted by the transformer for any given operating condition. Measurements of overall sound pressure level were not made, because excessive background noise in the room would mask the tone reduction. The average pure tone levels were therefore the only measures of stimulus strength taken during the experiment. These values are plotted along the abscissa. Open circles represent the no-cancellation condition; closed circles represent cancellation. Vertical bars depict one standard deviation of the relative annoyance judgments of the group. The parameter separating the functions in Figure $D_{-}-1$ is the operating voltage of the transformer. As can be seen from the figure, for the $120 \mathrm{~V}$ condition, the cancellation system produced an average reduction in sound level of $25.7 \mathrm{~dB}$ (across all three frequencies) and an average reduction in annoyance of 1.7 points. Likewise, for the $130 \mathrm{~V}$ condition, the cancellation system produced an average reduction in sound level of $19.3 \mathrm{~dB}$ and an average reduction in annoyance of 2.4 
points. The additional reduction in annoyance under the $130 \mathrm{~V}$ operating condition might be ascribed to the reduction of audible higher harmonic components (buzzing sounds) when the cancellation system was turned on. Unfortunatey, physical measurements of these higher harmonics were nat made during the experiment. In any case, the results of the experiment appear to show a reduction in subjective annoyance due to the transformer noise with the sound cancellation system in effect over the annoyance experienced without the cancellation system.

Any conclusions to be derived from this psychoacoustic demonstration experiment must be regarded with extreme caution. First, the group of listeners did not represent a random sample of naive observers. All members of the group were technical people concerned with the electric power industry. Second, the large standard deviations make the reduction in relative annoyance, with the sound cancellation system in operation, only a suggestive trend. The difference in annoyance ratings is not statistically significant (t-test, 0.05 level). Nevertheless, the functions for both operating voltages show the same trend. Third, physical measurements were not possible over the entire frequency range; therefore, the effect of the sound cancellation system on higher harmonjc components could nat be determined.

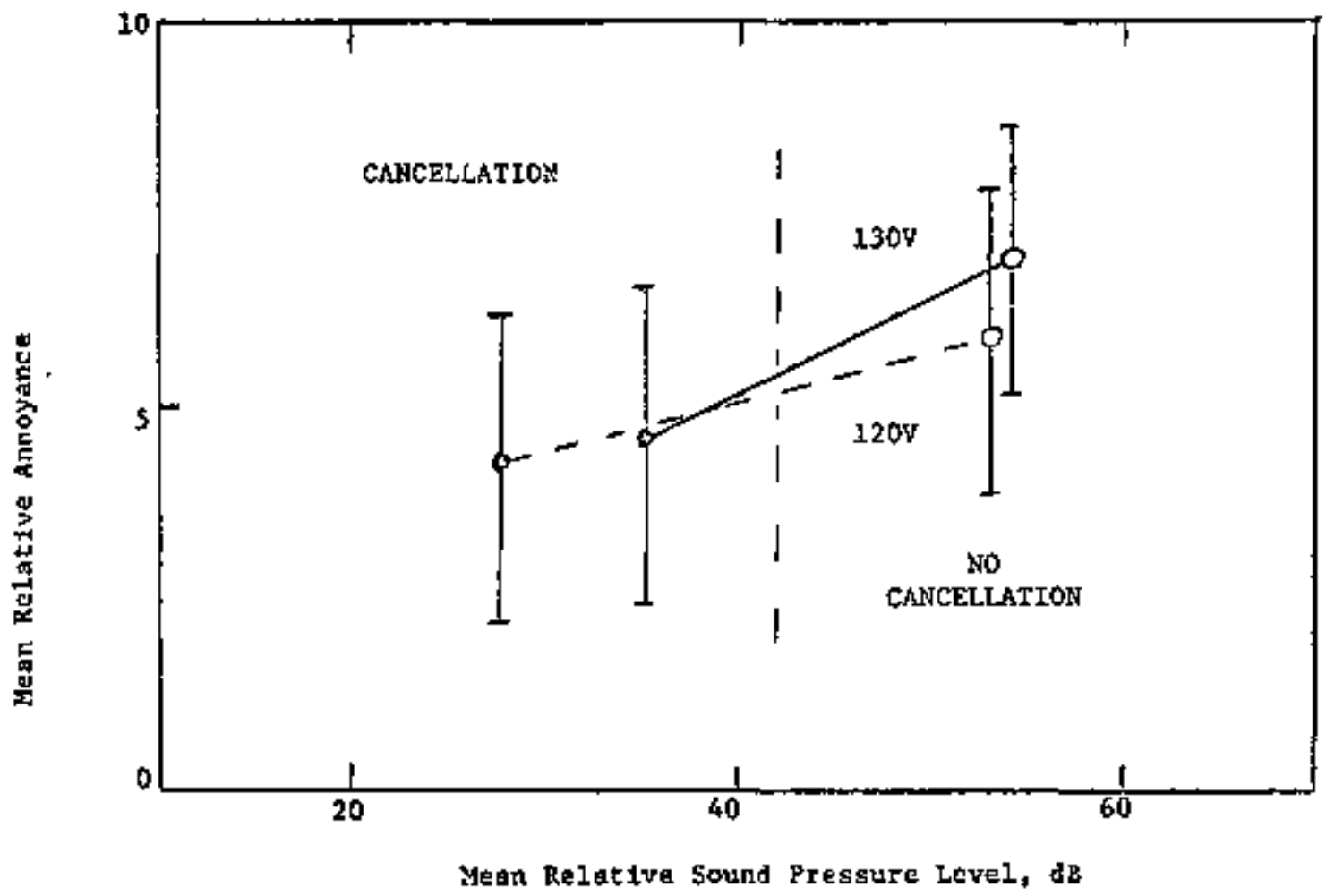

Figure D-1. Results of Psychoacoustic Demonstration Experiment 